$$
\begin{array}{r}
\text { MCS-P-452-0794 } \\
\text { OEIVED } \\
\text { NOV } 211996 \\
\text { OSTI }
\end{array}
$$

\title{
Tensor Methods for Large, Sparse Unconstrained Optimization *
}

\author{
Ali Bouaricha ${ }^{\dagger}$
}

\begin{abstract}
Tensor methods for unconstrained optimization were first introduced by Schnabel and Chow [SIAM J. Optimization, 1 (1991),-pp. 293-315], who describe these methods for small to moderate-size problems. The major contribution of this paper is the extension of these methods to large, sparse unconstrained optimization problems. This extension requires an entirely new way of solving the tensor model that makes the methods suitable for solving large, sparse optimization problems efficiently. We present test results for sets of problems where the Hessian at the minimizer is nonsingular and where it is singular. These results show that tensor methods are significantly more efficient and more reliable than standard methods based on Newton's method.
\end{abstract}

Key words, tensor methods, unconstrained optimization, sparse problems, large-scale optimization, singular problems

AMS(MOS) subject classification. $65 \mathrm{~K}$

\footnotetext{
- Part of this work was performed while the author was research associate at CERFACS (Centre Européen de Recherche et de Formation Avancée en (.alcul Scientifique).

'Mathematics and Computer Science Division, Argonne National Laboratory, Argonne, Mlinois, 60439. bouarichiamcs.anl.gov. This work was supported in part by the Office of Scientific Computing, U.S. Department of Energy, under Contract W-31-109-Eng-38.
} 


\section{DISCLAIMER}

Portions of this document may be illegible in electronic image products. Images are produced from the best available original document. 


\section{DISCLAIMER}

This report was prepared as an account of work sponsored by an agency of the United States Government. Neither the United States Government nor any agency thereof, nor any of their employees, makes any wartanty, express or implied, or assumes any legal liability or responsibility for the accuracy, completeness, or usefulness of any information, apparatus, product, or process disclosed, or represents that its use would not infringe privately owned rights. Reference herein to any specific commercial product, process, or service by trade name, trademark, manufacturer, or otherwise does not necessarily constitute or imply its endorsement, recommendation, or favoring by the United States Government or any agency thereof. The views and opinions of authors expressed herein do not necessarily state or reflect those of the United States Government or any agency thereof. 


\section{Introduction}

In this paper we describe tensor methods for solving the unconstrained optimization problem

$$
\text { given } f: \Re^{n} \rightarrow \Re \text {, find } x_{*} \in \Re^{n} \text { such that } f\left(x_{*}\right) \leq f(x) \text { for all } x \in D \text {, }
$$

where $D$ is some open set containing $x_{*}$. We assume that $f$ is at least twice continuously differentiable, and $n$ is large.

Tensor methods for unconstrained optimization are general-purpose methods primarily intended to improve upon the performance of standard methods, especially on problems where $\nabla^{2} f\left(x_{*}\right)$ has a șall rank deficiency. They are also intended to be at least as efficient as standard methods on problems where $\nabla^{2} f\left(x_{*}\right)$ is nonsingular.

Tensor methods for unconstrained optimization base each iteration upon the fourth-order model of the objective function $f(x)$,

$$
M_{T}\left(x_{c}+d\right)=f\left(x_{c}\right)+\nabla f\left(x_{c}\right) \cdot d+\frac{1}{2} \nabla^{2} f\left(x_{c}\right) \cdot d^{2}+\frac{1}{6} T_{c} \cdot d^{3}+\frac{1}{24} V_{c} \cdot d^{4},
$$

where $d \in \Re^{n}, x_{c}$ is the current iterate, $\nabla f\left(x_{c}\right)$ and $\nabla^{2} f\left(x_{c}\right)$ are the first and second analytic derivatives of $f$ at $x_{c}$, or finite difference approximations to them, and the tensor terms at $x_{c}$, $T_{c} \in \Re^{n \times n \times n}$ and $V_{c} \in \Re^{n \times n \times n \times n}$, are symmetric. (We use the notation $\nabla f\left(x_{c}\right) \cdot d$ for $\nabla f\left(x_{c}\right)^{T} d$, and $\nabla^{2} f\left(x_{c}\right) \cdot d^{2}$ for $d^{T} \nabla^{2} f\left(x_{c}\right) d$ to be consistent with the tensor notation $T_{c} \cdot d^{3}$ and $V_{c} \cdot d^{4}$. Also, for simplicity, we abbreviate terms of the form $d d, d d d$, and $d d d d$ by $d^{2}, d^{3}$, and $d^{4}$, respectively.) Before proceeding, we define the tensor notation used above.

Definition 1.1. Let $T \in \Re^{n \times n \times n}$. Then for $u, v, w \in \Re^{n}, T \cdot u v w \in \Re, T \cdot v w \in \Re^{n}$, with

$$
\begin{gathered}
T \cdot u v w=\sum_{i=1}^{n} \sum_{j=1}^{n} \sum_{k=1}^{n} T(i, j, k) u(i) v(j) w(k), \\
(T \cdot v w)(i)=\sum_{j=1}^{n} \sum_{k=1}^{n} T(i, j, k) v(j) w(k), \quad i=1, \ldots, n .
\end{gathered}
$$

Definition 1.2. Let $V \in \Re^{n \times n \times n \times n}$. Then for $r, u, v, w \in \Re^{n}, V \cdot r u v w \in \Re, V \cdot u v w \in \Re^{n}$ with

$$
\begin{gathered}
V \cdot \text { ruvw }=\sum_{i=1}^{n} \sum_{j=1}^{n} \sum_{k=1}^{n} \sum_{l=1}^{n} V(i, j, k, l) r(i) u(j) v(k) w(l), \\
(V \cdot u v w)(i)=\sum_{j=1}^{n} \sum_{k=1}^{n} \sum_{l=1}^{n} V(i, j, k, l) u(j) v(k) w(l), \quad i=1, \ldots, n .
\end{gathered}
$$

The tensor terms are selected so that the model interpolates a small number of function and gradient values from previous iterations. This results in $T_{c}$ and $V_{c}$ being low-rank tensors, which is crucial for the efficiency of the tensor method. The tensor method requires no more function or derivative evaluations per iteration and hardly more storage or arithmetic operations than does a standard method based on Newton's method.

Standard methods for solving unconstrained optimization problems are widely described in the literature; general references on this topic include Dennis and Schnabel [9], Fletcher [11], 
and Gill, Murray, and Wright [13]. In this paper, we propose extensions to standard methods that use analytic or finite-difference gradients and Hessians.

The standard method for unconstrained optimization, Newton's method, bases each iteration upon the quadratic model of $f(x)$,

$$
M_{N}\left(x_{c}+d\right)=f\left(x_{c}\right)+\nabla f\left(x_{c}\right) \cdot d+\frac{1}{2} \nabla^{2} f\left(x_{c}\right) \cdot d^{2}
$$

This method is defined when $\nabla^{2} f\left(x_{c}\right)$ is nonsingular and consists of setting the next iterate $x_{+}$ to the minimizer of (1.3), namely,

$$
x_{+}=x_{c}-\nabla^{2} f\left(x_{c}\right)^{-1} \nabla f\left(x_{c}\right) .
$$

A distinguishing feature of Newton's method is that if $\nabla^{2} f\left(x_{c}\right)$ is nonsingular at a local minimizer $x_{*}$, then the sequence of iterates produced by (1.4) converges quadratically to $x_{*}$. However, Newton's method is generally linearly convergent at best if $\nabla^{2} f\left(x_{*}\right)$ is singular [14].

Methods based on (1.2) have been shown to be more reliable and more efficient than standard methods on small to moderate-size problems [18]. In the test results obtained for both nonsingular and singular problems, the improvement by the tensor method over Newton's method is substantial, ranging from $30 \%$ to $50 \%$ in iterations and in function and derivative evaluations. Furthermore, the tensor method solves several problems that Newton's method fails to solve.

The tensor algorithms described in [18] are QR-based algorithms involving orthogonal transformations of the variable space. These algorithms are very effective for minimizing the tensor model when the Hessian is dense because they are very stable numerically, especially when the Hessian is singular. They are not efficient for sparse problems, however, because they destroy the sparsity of the Hessian due to the orthogonal transformation of the variable space. To preserve the sparsity of the Hessian, we have developed an entirely new way of solving the tensor model that employs a sparse variant of the Cholesky decomposition. This makes our new algorithms very well suited for sparse problems.

The remainder of this paper is organized as follows. In $\$ 2$ we briefly review the techniques introduced by Schnabel and Chow [18] to form the tensor model. In $\S 3$ we describe efficient algorithms for minimizing the tensor model when the Hessian is sparse. In $\S \S 4$ and 5 we discuss the globally convergent modifications for tensor methods for large, sparse unconstrained optimization. These consist of line search backtracking and model trust region techniques. A high-level implementation of the tensor method is given in $\S 6$. In $\S 7$ we describe comparative testing for an implementation based on the tensor method versus an implementation based on Newton's method. and we present summary statistics of the test results. Finally, in $\S 8$, we give a summary of our work and a discussion of future research.

\section{Forming the Tensor Model}

In this section, we briefly review the techniques that were introduced in [18] for forming the tensor model for unconstrained optimization.

As was stated in the preceding section, the tensor method for unconstrained optimization bases each iteration upon the fourth-order model of the nonlinear function $f(x)$ given by (1.2). 
The choices of $T_{c}$ and $V_{c}$ in (1.2) cause the third-order term $T_{c} \cdot d^{3}$ and the fourth-order term $V_{c} \cdot d^{4}$ to have simple and useful forms. These tensor terms are selected so that the tensor model interpolates function and gradient information at a set of $p$ not necessarily consecutive past iterates $x_{-1}, \ldots, x_{-p}$.

In the remainder of this paper, we restrict our attention to $p=1$. The reasons for this choice are that the performance of the tensor version that allows $p \geq 1$ is similar overall to that constraining $p$ to be 1 , and that the method is simpler and less expensive to implement in this case. (The derivation of the third- and fourth-order tensor terms for $p \geq 1$ is explained in detail in [18].)

The interpolation conditions at the past point $x_{-1}$ are given by

$$
f\left(x_{-1}\right)=f\left(x_{c}\right)+\nabla f\left(x_{c}\right) \cdot s+\frac{1}{2} \nabla^{2} f\left(x_{c}\right) \cdot s^{2}+\frac{1}{6} T_{c} \cdot s^{3}+\frac{1}{24} V_{c} \cdot s^{4}
$$

and

$$
\nabla f\left(x_{-1}\right)=\nabla f\left(x_{c}\right)+\nabla^{2} f\left(x_{c}\right) \cdot s+\frac{1}{2} T_{c} \cdot s^{2}+\frac{1}{6} V_{c} \cdot s^{3}
$$

where

$$
s=x_{-1}-x_{c} .
$$

Schnabel and Chow [18] choose $T_{c}$ and $V_{c}$ to satisfy (2.1) and (2.2). They first show that the interpolation conditions (2.1) and (2.2) uniquely determine $T_{c} \cdot s^{3}$ and $V_{c} \cdot s^{4}$. Multiplying $(2.2)$ by $s$ yields

$$
\nabla f\left(x_{-1}\right) \cdot s=\nabla f\left(x_{c}\right) \cdot s+\nabla^{2} f\left(x_{c}\right) \cdot s^{2}+\frac{1}{2} T_{c} \cdot s^{3}+\frac{1}{6} V_{c} \cdot s^{4} .
$$

Let $\alpha, \beta \in \Re$ be defined by

$$
\begin{aligned}
& \alpha=T_{c} \cdot s^{3}, \\
& \beta=V_{c} \cdot s^{4} .
\end{aligned}
$$

Then from (2.1) and (2.3) they obtain the following system of two linear equations in the two unknowns $\alpha$ and $\beta$ :

$$
\begin{aligned}
& \frac{1}{2} \alpha+\frac{1}{6} \beta=q_{1}, \\
& \frac{1}{6} \alpha+\frac{1}{24} \beta=q_{2},
\end{aligned}
$$

where $q_{1}, q_{2} \in \Re$ are defined by

$$
\begin{gathered}
q_{1}=\nabla f\left(x_{-1}\right) \cdot s-\nabla f\left(x_{c}\right) \cdot s-\nabla^{2} f\left(x_{c}\right) \cdot s^{2} \\
q_{2}=f\left(x_{-1}\right)-f\left(x_{c}\right)-\nabla f\left(x_{c}\right) \cdot s-\frac{1}{2} \nabla^{2} f\left(x_{c}\right) \cdot s^{2} .
\end{gathered}
$$

The system (2.4)-(2.5) is nonsingular; therefore the values of $\alpha$ and $\beta$ are uniquely determined. Hence, the interpolation conditions uniquely determine $T_{c} \cdot s^{3}$ and $V_{c} \cdot s^{4}$. Since these are the only interpolation conditions, the choice of $T_{c}$ and $V_{c}$ is vastly underdetermined. 
Schnabel and Chow [18] choose $T_{c}$ and $V_{c}$ by first selecting the smallest symmetric $V_{c}$, in the Frobenius norm, for which

$$
V_{c} \cdot s^{4}=\beta,
$$

where $\beta$ is determined by (2.4)-(2.5). Then they substitute this value of $V_{c}$ into (2.2), obtaining

$$
T_{c} \cdot s^{2}=a,
$$

where

$$
a=2\left(\nabla f\left(x_{-1}\right)-\nabla f\left(x_{c}\right)-\nabla^{2} f\left(x_{c}\right) \cdot s-\frac{1}{6} V_{c} \cdot s^{3}\right)
$$

This is a set of $n$ linear equations in $n^{3}$ unknowns $T_{c}(i, j, k), 1 \leq i, j, k \leq n$. Finally, Schnabel and Chow [18] choose the smallest symmetric $T_{c}$ and $V_{c}$, in the Frobenius norm, that satisfy the equations (2.6) $-(2.7)$. That is,

$$
\min _{V_{c} \in \Re^{n \times n \times n \times n}}\left\|V_{c}\right\|_{F}
$$

$$
\text { subject to } V_{c} \cdot s^{4}=\beta \text {, and } V_{c} \text { is symmetric, }
$$

and

$$
\min _{T_{c} \in \Re^{n \times n \times n}}\left\|T_{c}\right\|_{F}
$$

subject to $T_{c} \cdot s^{2}=a$, and $T_{c}$ is symmetric.

The solution to $(2.8)$ is

$$
V_{c}=\gamma(s \dot{\phi} \dot{\phi} s \dot{\phi}), \quad \gamma=\frac{\beta}{\left(s^{T} s\right)^{4}},
$$

where the tensor $V_{c}=s \dot{\phi} s \dot{\phi} s \dot{\phi} s \in \Re^{n \times n \times n \times n}$ is called a fourth-order rank-one tensor for which $V_{c}(i, j, k, l)=s(i) s(j) s(k) s(l), 1 \leq i, j, k, l \leq n$. (We use the notation $\dot{\otimes}$ to be consistent with [18].)

The solution to $(2.9)$ is

$$
T_{c}=b \dot{\otimes} \otimes s+s \otimes b \otimes s+s \otimes s \otimes b,
$$

where the notation $T=u \otimes v \otimes w, u, v, w \in \Re^{n}, T \in \Re^{n \times n \times n}$, is called a third-order rank-one tensor for which $T(i, j, k)=u(i) v(j) w(k)$. Here $b \in \Re^{n}$ is the unique vector for which $(2.10)$ satisfies (2.6). It is given by

$$
b=\frac{3 a\left(s^{T} s\right)-2 s\left(s^{T} a\right)}{3\left(s^{T} s\right)^{3}} .
$$

$T_{c}$ and $V_{c}$ determined by the minimum norm problems (2.9) and (2.8) have rank 2 and 1 , respectively. This is the key to form, store, and solve the tensor model efficiently. The whole process of forming the tensor model requires only $O\left(n^{2}\right)$ arithmetic operations. The storage needed for forming and storing the tensor model is only a total of $6 n$.

For further information we refer to [18]. 


\section{Solving the Tensor Model When the Hessian Is Sparse}

In this section we give algorithms for finding a minimizer of the tensor model (1.2) efficiently, when the Hessian is sparse.

The substitution of the values of $T_{c}$ and $V_{c}$ into (1.2) results in the tensor model

$$
M_{T}\left(x_{c}+d\right)=f\left(x_{c}\right)+\nabla f\left(x_{c}\right) \cdot d+\frac{1}{2} \nabla^{2} f\left(x_{c}\right) \cdot d^{2}+\frac{1}{2}\left(b^{T} d\right)\left(s^{T} d\right)^{2}+\frac{\gamma}{24}\left(s^{T} d\right)^{4} \text {. }
$$

As we stated in $\S 2$, we only consider the case $p=1$ where the tensor model interpolates $f(x)$ and $\nabla f(x)$ at the previous iterate. The generalization for $p \geq 1$ is fairly straightforward. This constraint is mainly motivated by our computational results. When we allow $p \geq 1$, our test results showed almost no improvement over the case where $p=1$. The tensor method is therefore considerably simpler, as well as cheaper in terms of storage and cost per iteration.

\subsection{Case 1: The Hessian Is Nonsingular}

We show that the minimization of (3.1) can be reduced to the solution of a third-order polynomial in one unknown, plus the solution of three systems of linear equations that all involve the same coefficient matrix $\nabla^{2} f\left(x_{c}\right)$. For conciseness, we use the notation $g=\nabla f\left(x_{c}\right)$ and $H=\nabla^{2} f\left(x_{c}\right)$.

A necessary condition for $d$ to be a local minimizer of (3.1) is that the derivative of the tensor model with respect to $d$ must be zero. That is,

$$
\nabla M_{T}\left(x_{c}+d\right)=g+H d+\left(b^{T} d\right)\left(s^{T} d\right) s+\frac{1}{2}\left(s^{T} d\right)^{2} b+\frac{\gamma}{6}\left(s^{T} d\right)^{3} s=0,
$$

which yields

$$
d=-H^{-1}\left(g+\left(b^{T} d\right)\left(s^{T} d\right) s+\frac{1}{2}\left(s^{T} d\right)^{2} b+\frac{\gamma}{6}\left(s^{T} d\right)^{3} s\right) .
$$

If we first premultiply the equation (3.2) by $s^{T}$ on both sides, we obtain a cubic equation (in $\beta$ ) in the unknowns $\beta=s^{T} d$ and $\theta=b^{T} d$,

$$
s^{T} H^{-1} g+\beta+s^{T} H^{-1} s \theta \beta+\frac{1}{2} s^{F} H^{-1} b \beta^{2}+\frac{\gamma}{6} s^{T} H^{-1} s \beta^{3}=0 .
$$

If we then premultiply the equation (3.2) by $b^{T}$ on both sides, we obtain another cubic equation (in $\beta$ ) in the unknowns $\beta$ and $\theta$,

$$
b^{T} H^{-1} g+\theta+b^{T} H^{-1} s \theta \beta+\frac{1}{2} b^{T} H^{-1} b \beta^{2}+\frac{\gamma}{6} b^{T} H^{-1} s \beta^{3}=0 .
$$

Thus, we obtain a system of two cubic equations in the two unknowns $\beta$ and $\theta$ which can be solved analytically.

We now show how to compute the solutions of this system of two cubic equations in two unknowns by computing the solutions of a single cubic equation in the unknown $\beta$. Let $u=$ $s^{T} H^{-1} g, v=s^{T} H^{-1} b, w=s^{T} H^{-1} s, y=b^{T} H^{-1} g$, and $z=b^{T} H^{-1} b$. We first calculate the value of $\theta$ as a function of $\beta$ using the equation (3.3):

$$
\theta=-\frac{\left(u+\beta+\frac{1}{2} v \beta^{2}+\frac{\gamma}{6} w \beta^{3}\right)}{w \beta} .
$$


Note that the denominator of (3.5) is equal to zero if either $\beta=0$ or $w=0$. We assume that $\beta \neq 0$; otherwise the tensor model would be reduced to the Newton model. Now, if $w=0$, then (3.3) would be quadratic in $\beta$. Therefore

$$
\beta=\frac{-1 \pm \sqrt{1-2 u v}}{2}
$$

Thus, real-valued minimizers of the tensor model (3.1) may exist only if $1-2 u v \geq 0$. It is easy to check that in order for $\theta$ to have a defined value, $1+v \beta$ cannot be zero.

If $\beta \neq 0$ and $w \neq 0$, we substitute the expression for $\theta$ into (3.4) and obtain

$$
-u+(y w-u v-1) \beta-\frac{3}{2} v \beta^{2}+\left(\frac{1}{2} w z-\frac{\gamma}{6} w-\frac{1}{2} v^{2}\right) \beta^{3}=0,
$$

which is a third-order polynomial in the one unknown $\beta$. The roots of (3.6) are computed analytically. We substitute the values of $\beta$ into (3.5) to calculate the values of $\theta$. Then we simply substitute the values of $\beta$ and $\theta$ into (3.2) to obtain the values of $d$. The major cost in this whole process is the calculation of $H^{-1} g, H^{-1} b$, and $H^{-1} s$.

After we compute the values of $d$, we determine which of them are potential minimizers. Our criterion is to select those values of $d$ that guarantee that there is a descent path from $x_{c}$ to $x_{c}+d$ for the model $M_{T}\left(x_{c}+d\right)$. Then among the selected steps, we choose the one that is closest to the current iterate $x_{c}$ in the Euclidean norm sense. If the tensor model has no minimizer, we use the standard Newton step as the step direction for the current iteration.

\subsection{Case 2: The Hessian Is Rank Deficient}

If the Hessian matrix is rank deficient, we transform the tensor model given in (3.1) by the following procedure. Let $d=\hat{d}+\delta$ for a fixed $\hat{d}$, where $\delta$ is the new unknown. Substituting this expression for $d$ into (3.1) yields the following tensor model, which is a function of $\delta$ :

$$
\begin{aligned}
M_{T}\left(x_{c}+d\right)= & f\left(x_{c}\right)+\nabla f\left(x_{c}\right) \cdot \hat{d}+\frac{1}{2} \nabla^{2} f\left(x_{c}\right) \cdot \hat{d}^{2}+\cdot \frac{1}{2}\left(b^{T} \hat{d}\right)\left(s^{T} \hat{d}\right)^{2} \\
& +\frac{\gamma}{24}\left(s^{T} \hat{d}\right)^{4}+\left(\nabla f\left(x_{c}\right)+\nabla^{2} f\left(x_{c}\right) \hat{d}+\left(b^{T} \hat{d}\right)\left(s^{T} \hat{d}\right) s\right. \\
& \left.+\frac{1}{2}\left(s^{T} \hat{d}\right)^{2} b+\frac{\gamma}{24}\left(s^{T} \hat{d}\right)^{3} s\right) \cdot \delta+\frac{1}{2}\left(\nabla^{2} f\left(x_{c}\right)\right. \\
& +\left(b^{T} \hat{d}+\frac{\gamma}{2} s s^{T}\right) \cdot \delta^{2}+\left(s^{T} \hat{d}\right)\left(b^{T} \delta\right)\left(s^{T} \delta\right)+\frac{1}{2}\left(b^{T} \delta\right)\left(s^{T} \delta\right)^{2} \\
& +\frac{\gamma}{6}\left(s^{T} \hat{d}\right)\left(s^{T} \delta\right)^{3}+\frac{\gamma}{24}\left(s^{T} \delta\right)^{4}
\end{aligned}
$$

If we let $\hat{\beta}=s^{T} \hat{d}, \hat{\theta}=b^{T} \hat{d}, \hat{g}=\nabla f\left(x_{c}\right)+\nabla^{2} f\left(x_{c}\right) \hat{d}+\hat{\theta} \hat{\beta} s+\frac{1}{2} \hat{\beta}^{2} b+\frac{\gamma}{6} \hat{\beta}^{3} s, c=b^{T} \hat{d}+\frac{\gamma}{2}$, and $\hat{H}=\nabla^{2} f\left(x_{c}\right)+c s s^{T}$, then we obtain the modified tensor model

$$
\begin{aligned}
M_{T}\left(x_{c}+d\right)= & M_{T}\left(x_{c}+\hat{d}\right)+\hat{g} \cdot \delta+\frac{1}{2} \hat{H} \cdot \delta^{2}+\hat{\beta}\left(b^{T} \delta\right)\left(s^{T} \delta\right) \\
& +\frac{1}{2}\left(b^{T} \delta\right)\left(s^{T} \delta\right)^{2}+\frac{\gamma}{6} \hat{\beta}\left(s^{T} \delta\right)^{3}+\frac{\gamma}{24}\left(s^{T} \delta\right)^{4}
\end{aligned}
$$


The advantage of this transformation is that the matrix $\hat{H}$ is likely to be nonsingular if the rank of $\left(\nabla^{2} f\left(x_{c}\right)\right)$ is at least $n-1$. A necessary and sufficient condition for $\hat{H}$ to be nonsingular is given in the following lemma. Let $g$ and $H$ denote $\nabla f\left(x_{c}\right)$ and $\nabla^{2} f\left(x_{c}\right)$, respectively.

Lemma 3.1. Let $H \in \Re^{n \times n}, s \in \Re^{n}$.

$$
H+c s s^{T} \text { is nonsingular if and only if } M=\left[\begin{array}{cc}
H & c s \\
c s^{T} & -c I
\end{array}\right] \text { is nonsingular. }
$$

(Note that the $\left[s^{T}-I\right]$ submatrix was premultiplied by the constant $c$ to symmetrize the augmented matrix $M$.)

Proof. We prove that there exists $v \in \Re^{n}, v \neq 0$, for which $\left(H+\operatorname{css}^{T}\right) v=0$, if and only if there exist $\bar{v} \in \Re^{n}, w \in \Re$, for which

$$
\left[\begin{array}{cc}
H & c s \\
c s^{T} & -c I
\end{array}\right]\left[\begin{array}{c}
\bar{v} \\
w
\end{array}\right]=\left[\begin{array}{l}
0 \\
0
\end{array}\right],\left[\begin{array}{l}
\bar{v} \\
w
\end{array}\right] \neq\left[\begin{array}{l}
0 \\
0
\end{array}\right] .
$$

Suppose first that $\left(H+c s s^{T}\right) v=0, v \neq 0$. Then for $\bar{v}=v, w=s^{T^{-}},(\bar{v}, w)$ satisfies (3.9). Conversely, if there exists $(\bar{v}, w)$ satisfying (3.9), then $s^{T} \bar{v}=w$, so $\left(H+c s s^{T}\right) \bar{v}=0$, and $\bar{v} \neq 0$; otherwise, $w=0$, which contradicts (3.9). Thus $\left(H+c s s^{T}\right)$ is singular if and only if $M$ is singular.

Corollary 3.2. Let $H \in \Re^{n \times n}, s \in \Re^{n}$.

$$
\text { If } H+c s s^{T} \text {. is nonsingular, then }\left[\begin{array}{ll}
H & c s
\end{array}\right] \text { has full row rank. }
$$

Proof. Follows from Lemma 3.1.

Lemma 3.3. Let $H \in \Re^{n \times n}, \operatorname{rank}(H)=n-1, s \in \Re^{n}$.

$$
H+\operatorname{css}^{T} \text { is nonsingular if and only if }\left[\begin{array}{ll}
H & \text { cs }
\end{array}\right] \text { has full row rank. }
$$

Proof. The only if part follows from Corollary 3.2. Now assume $\left[\begin{array}{ll}H & c s\end{array}\right]$ has full row rank. Since $H$ has rank $n-1, H=H_{1} H_{2}^{T}$, where $H_{1}, H_{2} \in \Re^{n \times(n-1)}$ have full column rank. Since $\left[\begin{array}{ll}H & c s\end{array}\right]$ has full row rank,

$$
\left(v^{T} H=0 \text { and } v^{T} s=0\right) \Rightarrow v=0
$$

From $H=H_{1} H_{2}{ }^{T}$ and the fact that $H_{2}$ has full column rank, (3.10) is equivalent to

$$
\left(v^{T} H_{1}=0 \text { and } v^{T} s=0\right) \Rightarrow v=0 .
$$


Thus the $n \times n$ matrix $\left[\begin{array}{ll}H_{1} & c s\end{array}\right]$ is nonsingular. Analogously, the $n \times n$ matrix $\left[\begin{array}{ll}H_{2} & s\end{array}\right]$ is nonsingular. Therefore

$$
\left[\begin{array}{ll}
H_{1} & c s
\end{array}\right]\left[\begin{array}{c}
H_{2}^{T} \\
s^{T}
\end{array}\right]=H_{1} H_{2}^{T}+c s s^{T}=H+c s s^{T}
$$

is nonsingular.

For $\delta$ to be a local minimizer of (3.8) the derivative of the tensor model (3.8) with respect to $\delta$ must be zero. That is,

$$
\begin{aligned}
\nabla M_{T}\left(x_{c}+\delta\right)= & \hat{g}+\hat{H} \delta+\hat{\beta}\left(s^{T} \delta\right) b+\hat{\beta}\left(b^{T} \delta\right) s+\left(s^{T} \delta\right)\left(b^{T} \delta\right) s \\
& +\left(\frac{1}{2} b+\frac{\gamma}{2} \hat{\beta} s\right)\left(s^{T} \delta\right)^{2}+\frac{\gamma}{6}\left(s^{T} \delta\right)^{3} s=0
\end{aligned}
$$

which yields

$$
\begin{aligned}
\delta= & -\hat{H}^{-1}\left(\hat{g}+\hat{\beta}\left(s^{T} \delta\right) b+\hat{\beta}\left(b^{T} \delta\right) s+\left(s^{T} \delta\right)\left(b^{T} \delta\right) s\right. \\
& \left.+\left(\frac{1}{2} b+\frac{\gamma}{2} \hat{\beta} s\right)\left(s^{T} \delta\right)^{2}+\frac{\gamma}{6}\left(s^{T} \delta\right)^{3} s\right) .
\end{aligned}
$$

Premultiplying (3.12) by $s^{T}$ on both sides results in a cubic equation (in $\beta$ ) in the two unknowns $\beta=s^{T} \delta$ and $\theta=b^{T} \delta$ :

$$
\begin{aligned}
& s^{T} \hat{H}^{-1} \hat{g}+\left(1+\hat{\beta} s^{T} \hat{H}^{-1} b\right) \beta+\hat{\beta} s^{T} \hat{H}^{-1} s \theta+s^{T} \hat{H}^{-1} s \beta \theta \\
& +\left(\frac{1}{2} s^{T} \hat{H}^{-1} b+\frac{\gamma}{2} \hat{\beta} s^{T} \hat{H}^{-1} s\right) \beta^{2}+\frac{\gamma}{6} s^{T} \hat{H}^{-1} s \beta^{3}=0 .
\end{aligned}
$$

The premultiplication of (3.12) by $b^{T}$ on both sides yields another cubic equation (in $\beta$ ) in the two unknowns $\beta$ and $\theta$ :

$$
\begin{aligned}
& b^{T} \hat{H}^{-1} \hat{g}+\left(1+\hat{\beta} b^{T} \hat{H}^{-1} s\right) \theta+\hat{\beta} b^{T} \hat{H}^{-1} b \beta+b^{T} \hat{H}^{-1} s \beta \theta \\
& +\left(\frac{1}{2} b^{T} \hat{H}^{-1} b+\frac{\gamma}{2} \hat{\beta} b^{T} \hat{H}^{-1} s\right) \beta^{2}+\frac{\gamma}{6} b^{T} \hat{H}^{-1} s \beta^{3}=0 .
\end{aligned}
$$

Therefore, we obtain a system of two cubic equations in the two unknowns $\beta$ and $\theta$, which we can solve analytically.

Since (3.13) is linear in $\theta$, we can compute $\theta$ as a function of $\beta$ and then substitute its expression into (3.14) to obtain an equation in the one unknown $\beta$. Let $u=s^{T} \hat{H}^{-1} \hat{g}, v=$ $s^{T} \hat{H}^{-1} b, w=s^{T} \hat{H}^{-1} s, y=b^{T} \hat{H}^{-1} \hat{g}$, and $z=b^{T} \hat{H}^{-1} b$. Equation (3.13) yields 


$$
\begin{aligned}
\theta= & \frac{1}{w(\hat{\beta}+\beta)}\left(y w \hat{\beta}-u-u v \hat{\beta}+\left(y w+z w \hat{\beta}^{2}-2 v \hat{\beta}-v^{2} \hat{\beta}^{2}-u v-1\right) \beta\right. \\
& \left.+\left(\frac{3}{2} z w \hat{\beta}-\frac{\gamma}{2} w \hat{\beta}-\frac{3}{2} v-\frac{3}{2} v^{2} \hat{\beta}\right)+\left(\frac{1}{2} z w-\frac{\gamma}{6} w-\frac{v^{2}}{2}\right) \beta^{3}\right) .
\end{aligned}
$$

The denominator of (3.15) is equal to zero if either $\hat{\beta}+\beta=0$ or $w=0$. If $w=0$, then (3.13) would be quadratic in $\beta$. Therefore

$$
\beta=\frac{-(1+\hat{\beta} v) \pm \sqrt{(1+\hat{\beta} v)^{2}-2 u v}}{v}
$$

Hence, real-valued minimizers of the tensor model (3.8) may exist only if $(1+\hat{\beta} v)^{2} \geq 2 u v$ and $v \neq 0$. It is straightforward to verify from (3.14) that for $\theta$ to be defined $(\hat{\beta}+\beta) v$ cannot equal -1 . Now, if $\hat{\beta}+\beta=0$, then (3.13) reduces to the following cubic equation in $\beta$ :

$$
u+(1+\hat{\beta} v) \beta+\left(\frac{1}{2} v+\frac{\gamma}{2} w \hat{\beta}\right) \beta^{2}+\frac{\gamma}{6} w \beta^{3}=0
$$

Once we calculated the expressions for $\beta$ from (3.16), we substitute them into the following equation for $\theta$ obtained from (3.14):

$$
\theta=-y-z \hat{\beta} \beta-\left(\frac{1}{2} z+\frac{\gamma}{2} v \hat{\beta}\right) \beta^{2}-\frac{\gamma}{6} v \beta^{3} .
$$

If neither $\hat{\beta}+\beta=0$ nor $w=0$, we substitute the expression (3.15) into (3.14) and obtain

$$
\begin{aligned}
& -\left(u+2 \hat{\beta} v+\hat{\beta} u v+\hat{\beta}^{2} v^{2}+1\right)+\left(y w+\hat{\beta}^{2} z w-\hat{\beta} v-v-u v\right) \beta \\
& +\left(\hat{\beta}^{2} z w+\frac{1}{2} \hat{\beta} z w-\frac{1}{2} v-\frac{\gamma}{2} \hat{\beta} w-\frac{1}{2} \hat{\beta} v^{2}\right) \beta^{2}+\left(\frac{1}{2} z w-\frac{\gamma}{6} w-\frac{1}{2} v^{2}\right) \beta^{3}=0,
\end{aligned}
$$

hich is a third-order polynomial in the one unknown $\beta$. The roots of (3.17) are then computed analytically. After we determine the values of $\beta$, we substitute them into (3.15) to calculate the corresponding values of $\theta$. Then, we simply substitute the values of $\beta$ and $\theta$ into (3.12) to obtain the values of $\delta$. The dominant cost in this whole process is the computation of $\hat{H}^{-1} \hat{g}$, $\hat{H}^{-1} b$, and $\hat{H}^{-1}$ s.

Similar to the nonsingular case, a minimizer $\delta$ is selected such that there exists a descent path from the current point $x_{c}$ to $x_{c}+\delta$, and that $\delta$ is closest to $x_{c}$ in the Euclidean norm sense.

To obtain the tensor step $d$, we set $d$ to $\hat{d}+\delta$. An appropriate choice of $\hat{d}$ is the step used in the previous iteration simply because it has the right scale.

To solve linear systems of the form $\hat{H} x=b$, where $\hat{H}=H+\operatorname{css}^{T}, H \in \Re^{n \times n}$ sparse and $s \in \Re^{n}$ full, we use the augmented matrix $M$ defined in Lemma 3.1. That is, we write $\left(H+c s s^{T}\right) x=b$ as

$$
\left[\begin{array}{cc}
H & c s \\
c s^{T} & -c I
\end{array}\right]\left[\begin{array}{l}
x \\
w
\end{array}\right]=\left[\begin{array}{l}
b \\
0
\end{array}\right]
$$


The $(n+1) \times(n+1)$ matrix in (3.18) is sparse and can be factored efficiently as long as the last row and column are not pivoted until the last few iterations. In fact, we can combine the nonsingular and singular cases by factoring $H$, but we shift to a factorization of the augmented matrix if $H$ is discovered to be singular with rank $n-1$. However, we use a Schur complement method to obtain the solution of the augmented matrix by updating the solution from the system $H x=b$. This choice was motivated by the fact that the Schur complement method is simpler and more convenient to use than the factorization of the augmented matrix $M$. We describe this updating scheme in $\S 6$.

If the Schur complement method shows that $M$ is rank deficient (a case that is very rare in practice), or $\mathbb{H}$ has rank less than $n-1$, we use the standard Newton step as the step direction for the current iteration.

\section{Line Search Backtracking Techniques}

The line search global strategy we use in conjunction with our tensor method for large, sparse unconstrained optimization is similar to the one used for nonlinear equations $[4,6]$. This strategy has shown to be very successful for large, sparse systems of nonlinear equations. We also found that it is superior to the approach used by Schnabel and Chow [18]. The main difference between the two approaches is that ours always tries the full tensor step first. If this provides enough decrease in the objective function, then we terminate; otherwise we find acceptable next iterates in both the Newton and tensor directions and select the one with the lower function value as the next iterate. Schnabel and Chow, on the other hand, always find acceptable next iterates in both the Newton and tensor directions and choose the one with the lower function value as the next iterate. In practice, our approach almost always requires fewer function evaluations while retaining the same efficiency in iteration numbers. The global framework for line search methods for unconstrained minimization is given in Algorithm 4.1.

Algorithm 4.1. (ilobal Framework for Line Search Methods for Unconstrained Minimization

Let $x_{c}$ be the current iterate,

$d_{t}$ the tensor step,

$d_{n}$ is the Newton step,

$g=\nabla f\left(x_{c}\right)$,

$f_{c}=f\left(x_{c}\right)$,

slope $=g^{T} d_{t}$

and $\alpha=10^{-4}$.

$x_{+}^{t}=x_{c}+d_{t}$

$f_{p}=f\left(x_{+}^{t}\right)$

if (minimizer of the tensor model was found) then

if $f_{p}<f_{c}+\alpha \cdot$ slope then

else

$$
x_{+}=x_{+}^{t}
$$

Find an acceptable $x_{+}^{n}$ in the Newton direction $d_{n}$ using Algorithm A6.3.1 [9, p.325] 
Find an acceptable $x_{+}^{t}$ in the tensor direction $d_{t}$ using Algorithm A6.3.1 [9, p.325]

$$
\begin{aligned}
& \text { if } f\left(x_{+}^{n}\right)<f\left(x_{+}^{t}\right) \text { then } \\
& x_{+}=x_{+}^{n} \\
& \text { else } \\
& \quad x_{+}=x_{+}^{t} \\
& \text { endif } \\
& \text { endif }
\end{aligned}
$$

else

Find an acceptable $x_{+}^{n}$ in the Newton direction $d_{n}$ using Algorithm A6.3.1 [9, p.325]

endif

$x_{+}=x_{+}^{n}$

\section{Model Trust Region Techniques}

The two computational methods-the locally constrained optimal (or "hook") step and the dogleg step-are generally used for approximately solving the trust region problem based on the standard model,

$$
\begin{gathered}
\text { minimize } f\left(x_{c}\right)+\nabla f\left(x_{c}\right) \cdot d+\frac{1}{2} \nabla^{2} f\left(x_{c}\right) \cdot d^{2} \\
\text { subject to }\|d\|_{2} \leq \delta_{c},
\end{gathered}
$$

where $\delta_{c}$ is the current trust region radius. When $\delta_{c}$ is shorter than the Newton step, the locally constrained optimal step [16] finds a $\mu_{c}$ such that $\left\|d\left(\mu_{c}\right)\right\|_{2} \approx \delta_{c}$, where $d\left(\mu_{c}\right)=$ $-\left(\nabla^{2} f\left(x_{c}\right)+\mu I\right)^{-1} \nabla f\left(x_{c}\right)$. Then it takes $x_{+}=x_{c}+d\left(\mu_{c}\right)$. The dogleg step is a modification of the trust region algorithm introduced by Powell [17]. However, rather than finding a point $x_{+}=x_{c}+d\left(\mu_{c}\right)$ on the curve $d\left(\mu_{c}\right)$ such that $\left\|x_{+}-x_{c}\right\| \approx \delta_{c}$, it approximates this curve by a piecewise linear function in the subspace spanned by the Newton step and the steepest descent direction $-\nabla f\left(x_{c}\right)$, and takes $x_{+}$as the point on this approximation for which $\left\|x_{+}-x_{c}\right\|=\delta_{c}$. (See, e.g., [9] for more details.)

Unfortunately these two methods are hard to extend to the tensor model, which is a fourthorder model. Trust region algorithms based on (5.19) are well defined because it is always possible to find a unique point $x_{+}$on the curve such that $\left\|x_{+}-x_{c}\right\|=\delta_{c}$. Additionally, the value of $f\left(x_{c}\right)+\nabla f\left(x_{c}\right) \cdot d+\frac{1}{2} \nabla^{2} f\left(x_{c}\right) \cdot d^{2}$ along the curve $d\left(\mu_{c}\right)$ is monotonically decreasing from $x_{c}$ to $x_{+}^{n}$, where $x_{+}^{n}=x_{c}+d_{n}$, which inakes the process reasonable. These properties do not extend to the tensor model, which is a fourth-order model that may not be convex. Furthermore, the analogous curve to $d\left(\mu_{c}\right)$ is more expensive to compute. For these reasons, we consider a different trust region approach for our tensor methods.

The trust region approach that is discussed in this section is a two-dimensional trust region step over the subspace spanned by the steepest descent direction and the tensor (or standard) step. The main reasons that lead us to adopt this approach are that it is easy to construct, closely related to dogleg type algorithms over the same subspace. This step may be close to optimal trust region step algorithms in practice. Byrd, Schnabel, and Shultz [7] have shown that for 
unconstrained optimization using a standard quadratic model, the analogous two-dimensional minimization approach produces nearly as much decrease in the quadratic model as the optimal trust region step in almost all cases.

The two-dimensional trust region approach for the tensor model computes an approximate solution to

$$
\begin{gathered}
\operatorname{minimize} f\left(x_{c}\right)+\nabla f\left(x_{c}\right) \cdot d+\frac{1}{2} \nabla^{2} f\left(x_{c}\right) \cdot d^{2}+\frac{1}{2}\left(b^{T} d\right)\left(s^{T} d\right)^{2}+\frac{\gamma}{24}\left(s^{T} d\right)^{4} \\
\text { subject to }\|d\|_{2} \leq \delta_{c},
\end{gathered}
$$

by performing a two-dimensional minimization,

$$
\begin{gathered}
\operatorname{minimize} f\left(x_{c}\right)+\nabla f\left(x_{c}\right) \cdot d+\frac{1}{2} \nabla^{2} f\left(x_{c}\right) \cdot d^{2}+\frac{1}{2}\left(b^{T} d\right)\left(s^{T} d\right)^{2}+\frac{\gamma}{24}\left(s^{T} d\right)^{4} \\
\text { subject to }\|d\|_{2} \leq \delta_{c}, \quad d \in\left[d_{t}, g_{s}\right]
\end{gathered}
$$

where $d_{t}$ and $g_{s}$ are the tensor step and the steepest descent direction, respectively, and $\delta_{c}$ is the trust region radius. This approach will always produce a step that reduces the quadratic model by at least as much as a dogleg-type algorithm, which reduces $d$ to a piecewise linear curve in the same subspace. At each iteration of the tensor algorithm, the trust region method either solves (5.20) or minimizes the standard linear model over the two-dimensional subspace spanned by the standard Newton step and the steepest descent direction. The decision of whether to use the tensor or standard model is made using the following criterion:

if (no minimizer of the tensor model was found) or $\left(\nabla f\left(x_{c}\right)^{T} d_{t}>-10^{-4}\left\|\nabla f\left(x_{c}\right)\right\|_{2}\left\|d_{t}\right\|_{2}\right)$ then

$$
x_{+}=x_{c}+\alpha d_{n}-\beta g_{s} ; \alpha, \beta \text { selected by trust region algorithm }
$$

$x_{+}=x_{c}+\alpha d_{t}-\beta g_{s} ; \alpha, \beta$ selected by trust region algorithm endif

Before we define the two-dimensional trust region step for tensor methods, we show how to convert the problem

$$
\begin{gathered}
\text { minimize } f\left(x_{c}\right)+\nabla f\left(x_{c}\right) \cdot d+\frac{1}{2} \nabla^{2} f\left(x_{c}\right) \cdot d^{2}+\frac{1}{2}\left(b^{T} d\right)\left(s^{T} d\right)^{2}+\frac{\gamma}{24}\left(s^{T} d\right)^{4} \\
\text { subject to }\|d\|_{2}=\delta_{c}, \quad d \in\left[d_{t}, g_{s}\right],
\end{gathered}
$$

to an unconstrained minimization problem.

First, we make $g_{s}$ orthogonal to $d_{t}$ by performing the Householder transformation:

$$
\hat{g_{s}}=g_{s}-d_{t} \frac{g_{s}^{T} d_{t}}{d_{t}^{T} d_{t}}
$$

then, we normalize both $\hat{g}_{s}$ and $d_{t}$ to obtain

$$
\tilde{d}_{t}=\frac{d_{t}}{\left\|d_{t}\right\|_{2}},
$$




$$
\tilde{g_{s}}=\frac{\hat{g_{s}}}{\left\|\hat{g_{s}}\right\|_{2}}
$$

Since $d$ is in the subspace spanned by the tensor step $\tilde{d}_{t}$ and the steepest descent direction $\tilde{g}_{s}$, it can be written as

$$
d=\alpha \tilde{d}_{t}+\beta \tilde{g}_{s}, \quad \alpha, \beta \in \Re .
$$

If we square the $l_{2}$ norm of this expression for $d$ and set it to $\delta^{2}$, we obtain the following equation for $\beta$ as a function of $\alpha$

$$
\beta=\sqrt{\delta^{2}-\alpha^{2}} \text {. }
$$

Substituting this expression for $\beta$ into (5.25) and then the resulting $d$ into (5.21) yields the global minimization problem in the one variable $\alpha$, given by (5.26) below. Thus, problems (5.26) and (5.21) are equivalent. Let $g_{h g}=\tilde{g}_{s}^{T} H \tilde{g}_{s}, d_{h d}=\tilde{d}_{t}^{T} H \tilde{d}_{t}, d_{h g}=\tilde{d}_{t}^{T} H \tilde{g}_{s}, b_{t}=b^{T} \tilde{d}_{t}, s_{t}=s^{T} \tilde{d}_{t}$, $b_{g}=b^{T} \tilde{g}_{s}$, and $s_{g}=s^{T} \tilde{g}_{s}$.

$$
\text { minimize } \begin{aligned}
f\left(x_{c}\right) & +\frac{1}{2} \delta_{c}^{2} g_{h g}+\frac{\gamma}{24} \delta_{c}^{4} s_{g}^{4}+\left(1+\delta_{c}^{2} b_{g} s_{g}^{2}\right) \sqrt{\delta_{c}^{2}-\alpha^{2}} \\
& +\left(d_{h g}+\frac{\gamma}{6} \delta_{c}^{2} s_{t} s_{g}^{3}\right) \alpha \sqrt{\delta_{c}^{2}-\alpha^{2}}+\left(b_{t} s_{g} s_{t}+b_{g} s_{t}^{2}+b_{t} s_{t} s_{g}\right. \\
& \left.-b_{g} s_{g}^{2}\right) \alpha^{2} \sqrt{\delta_{c}^{2}-\alpha^{2}}+\left(\delta_{c}^{2} b_{g} s_{g} s_{t}+\delta_{c}^{2} b_{t} s_{g}^{2}+\delta_{c}^{2} b_{g} s_{t} s_{g}\right) \alpha \\
& +\left(\frac{1}{2} d_{h d}-\frac{1}{2} g_{h g}+\frac{1}{2} b_{t} s_{t}^{2}+\frac{\gamma}{4} \delta_{c}^{2} s_{t}^{2} s_{g}^{2}-\frac{\gamma}{12} \delta_{c}^{2} s_{g}^{4}\right) \alpha^{2} \\
& -\left(b_{g} s_{g} s_{t}+b_{t} s_{g}^{2}+b_{g} s_{t} s_{g}\right) \alpha^{3}+\left(\frac{\gamma}{24} s_{t}^{4}-\frac{\gamma}{4} s_{t}^{2} s_{g}^{2}+\frac{\gamma}{24} s_{g}^{4}\right) \alpha^{4} \\
& +\left(\frac{\gamma}{6} s_{t}^{3} s_{g}-\frac{\gamma}{6} s_{t} s_{g}^{3}\right) \alpha^{3} \sqrt{\delta_{c}^{2}-\alpha^{2}}
\end{aligned}
$$

where $-\delta_{c}<\alpha<\delta_{c}$.

To transform the problem

$$
\begin{gathered}
\operatorname{minimize} f\left(x_{c}\right)^{-}+\nabla f\left(x_{c}\right) \cdot d+\frac{1}{2} \nabla^{2} f\left(x_{c}\right) \cdot d^{2} \\
\text { subject to }\|d\|_{2}=\delta_{c}, \quad d \in\left[d_{n}, g\right]
\end{gathered}
$$

to an unconstrained minimization problem, we use the same procedure described above to show that $(5.27)$ is equivalent to the following global minimization problem in the one variable $\alpha$ :

$$
\text { minimize } f\left(x_{c}\right)+\frac{1}{2} \delta_{c}^{2} g_{h g}+\sqrt{\delta_{c}^{2}-\alpha^{2}}+d_{h g} \alpha \sqrt{\delta_{c}^{2}-\alpha^{2}}+\left(\frac{1}{2} d_{h d}-\frac{1}{2} g_{h g}\right) \alpha^{2}
$$

where $-\delta_{c}<\alpha<\delta_{c}$.

Algorithm 5.1. Two-Dimensional Trust Region for Tensor Methods

Let $d_{t}$ be the tensor step,

$d_{n}$ the standard step,

$x_{c}$ the current iterate, 
$f_{c}=f\left(x_{c}\right)$,

$x_{+}$the next iterate,

$f_{+}=f\left(x_{+}\right)$,

$g_{s}=-\nabla f\left(x_{c}\right)$, the steepest descent direction,

$g_{c}=\nabla f\left(x_{c}\right)$,

$H_{c}=\nabla^{2} f\left(x_{c}\right)$,

and $\delta_{c}$ the current trust region radius.

$\tilde{d}_{t}, \tilde{g}_{s}$ are given by (5.23) and (5.24), respectively.

$\tilde{d}_{n}$ is obtained in an analogous way to $\tilde{d}_{t}$; by applying transformations (5.22) and (5.23) to it.

1. if tensor model selected then

Solve problem (5.26) using the procedure described in Algorithm 3.4 [6]

else \{standard Newton model selected\}

Solve problem (5.28) using the procedure described in Algorithm 3.4 [6] endif

2. if tensor model selected then

$d=\alpha_{*} \tilde{d}_{t}+\tilde{g}_{s} \sqrt{\delta_{c}^{2}-\alpha_{*}^{2}}$

where $\alpha_{*}$ is the global minimizer of (5.26)

else \{standard Newton model selected\}

$d=\alpha_{*} \tilde{d}_{n}+\tilde{g}_{s} \sqrt{\delta_{c}^{2}-\alpha_{*}^{2}}$

where $\alpha_{*}$ is the global minimizer of (5.28)

endif

3. \{ Check new iterate and update trust region radius.

$x_{+}=x_{c}+d$

if $\frac{f_{+}-f_{c}}{\text { pred }} \geq 10^{-4}$ then

the global step $d$ is successful

else

decrease trust region

go to step 1

endif

where

pred $=\left(f_{c}+g_{c} \cdot d+\frac{1}{2} H_{c} \cdot d^{2}+\frac{1}{2}\left(b^{T} d\right)\left(s^{T} d\right)^{2}+\frac{\gamma}{24}\left(s^{T} d\right)^{4}\right)-f_{c}$, if tensor model selected,

pred $=\left(f_{c}+g_{c} \cdot d+\frac{1}{2} H_{c} \cdot d^{2}\right)-f_{c}$, if standard Newton model selected.

The methods used for adjusting the trust radius during and between steps are given in Algorithm A6.4.5 [9, p.338]. The initial trust radius can be supplied by the user; if not, it is set to the length of the initial Cauchy step.

\section{A High-Level Algorithm for the Tensor Method}

In this section, we present the overall algorithm for the tensor method for large, sparse unconstrained optimization. Algorithm 6.1 is a high-level description of an iteration of the tensor 
method that was described in $\S \S 3-5$. A summary of the test results for this implementation is presented in $\$ 7$.

Algorithm 6.1. An Iteration of the Tensor Method for Large, Sparse Unconstrained Optimization

Let $x_{c}$ be the current iterate,

$d_{t}$ the tensor step,

and $d_{n}$ the Newton step.

1. Calculate $\nabla f\left(x_{c}\right)$ and decide whether to stop. If not:

2. Calculate $\nabla^{2} f\left(x_{c}\right)$.

3. Calculate the terms $T_{c}$ and $V_{c}$ in the tensor model, so that the tensor model interpolates $f(x)$ and $\nabla f(x)$ at the past point.

4. Find a potential minimizer $d_{t}$ of the tensor model (3.1).

5. Find an acceptable next iterate $x_{+}$using either a line search or a two-dimensional trust region global strategy.

6. $x_{c}=x_{+}$,

$f\left(x_{c}\right)=f\left(x_{+}\right)$,

go to step 1 .

In step 1, the gradient is either computed analytically or approximated by the algorithm A5.6.3 given in Dennis and Schnabel [9]. In step 2, the Hessian matrix is either calculated analytically or approximated by a graph coloring algorithm described in [8]. Note that it is crucial to supply an analytic gradient if the finite difference Hessian matrix requires many gradient evaluations. Otherwise, the methods described in this paper may not be practical, and inexact type of methods may be preferable. The procedures for calculating $T_{c}$ and $V_{c}$ in step 3 were discussed in $\S 2$. Step 4 calculates $d_{t}$ as described in $\S \S 3-4$. The Newton step $d_{n 2}$ is also computed as a by-product of the minimization of the tensor model. The Newton step $d_{n}$ is the modified Vewton step $\left(\nabla^{2} f\left(x_{c}\right)+\mu I\right)^{-1} \nabla f\left(x_{c}\right)$, where $\mu=\theta$ if $\nabla^{2} f\left(x_{c}\right)$ is safely positive definite, and $\mu>0$ otherwise. To obtain the perturbation $\mu$, we use a modification of MA27 [10] advocated by Gill, Murray, Ponceleon, and Saunders in [12]. In this method we first compute the $L D L^{T}$ of the Hessian matrix using the MA27 package, then change the block diagonal matrix $D$ to $D+E$. The modified matrix is block diagonal positive definite. This guarantees that the decomposition $L(D+E) L^{T}$ is positive definite as well. Note that the Hessian matrix is not modified if it is already positive definite.

The tensor and Newton algorithms terminate if $\left\|\nabla f\left(x_{c}\right)\right\|_{2} \leq 10^{-5}$ or $\|d\|_{2}<10^{-9}$.

Another implementation issue that deserves some attention is how to find a solution to the augmented system (3.18) when the Hessian matrix is rank deficient. To do this, we use a Schur complement method to update the solution $x$ obtained from solving $H x=b$. This requires that $H$ must have full rank. Thus, some modifications are necessary in order for this method to work. We have modified the factorization phase of MA27 to be able to detect the row and column indices of the first pivot that is less or equal than some given tolerance tol. Note that if the rank of the Hessian matrix is less than $n-1$, then we skip this whole updating scheme and perturb the matrix as described in the preceding paragraph. We also modified the solve 
phase of MA27 such that whenever there is a zero pivot, the corresponding solution component is set to zero. This way the solution of $H x=b$ is the same as the solution of $H_{e} y=b$ (where $H_{e}$ is the matrix $H$ minus the row and column at which singularity occurred. Since $y$ has $n-1$ components, the remaining one, which is also the component corresponding to the zero pivot, is set to 0 ). Afterwards, we obtain the solution of an augmented system using a Schur complement method, where the coefficient matrix is the matrix $H$ augmented by two rows and columns; that is, the $(n+1)$-st row and column are the ones at which singularity was detected, and the $(n+2)$-nd row and column are $c s^{T}$ and $c s$, respectively. The Schur complement method is implemented by first invoking MA39AD [1] to form the Schur complement $S=D-C H^{-1} B$ of $H$ in the extended matrix, where $D$ is the 2 by 2 lower right submatrix, $C$ is the lower left 2 by $n$ submatrix, and $B$ is the upper right $n$ by 2 submatrix, of the augmented matrix. The Schur complement is then factored into its QR factors. Next, MA39BD [1] solves the extended system (3.18) using the following well-known scheme:

1. Solve $H u=b$, for $u$.

2. Solve $S y=b-C^{\prime} u$, for $y$.

3. Solve $H v=B y$, for $v$.

4. $x=u-v$.

\section{Test Results}

We tested our tensor and Newton algorithms on a variety of nonsingular-and singular test problems. In the following we present and discuss summary statistics of the test results.

All our computations were performed on a Sun Sparc 10 Model 40 machine using doubleprecision arithmetic.

First, we tested our program on the set of unconstrained optimization problems from the CUTE [3] and the MINPACK-2 [2] collections. Most of these problems have nonsingular Hessians at the solution. We also created singular test problems as proposed in $[4,19]$ by modifying the nonsingular test problems from the CUTE collection as follows. Let

$$
f(x)=\sum_{i=1}^{m} f_{i}^{2}(x)
$$

be the function to minimize, where $f_{i}: \Re^{n} \rightarrow \Re$ and $m$ is the number of element functions, and

$$
F^{T}(x)=\left(f_{1}(x), \ldots, f_{m}(x)\right) .
$$

In many cases, $F(x)=0$ at the minimizer $x_{*}$, and $F^{\prime}\left(x_{*}\right)$ is nonsingular. Then according to $[4,19]$, we can create singular systems of nonlinear equations from $(7.1)$ by forming

$$
\hat{F}(x)=F(x)-F^{\prime}\left(x_{*}\right) A\left(A^{T} A\right)^{-1} A^{T}\left(x-x_{*}\right),
$$

where $A \in \Re^{n \times k}$ has full column rank with $1 \leq k \leq n$. Hence, $\hat{F}\left(x_{*}\right)=0$ and $\hat{F}^{\prime}\left(x_{*}\right)$ has rank $n-k$. For unconstrained optimization, we simply need to define the singular function 


$$
\hat{f}(x)=\frac{1}{2} \hat{F}(x)^{T} \hat{F}(x) .
$$

From (7.3) and $\hat{F}\left(x_{*}\right)=0$, we obtain $\nabla \hat{f}\left(x_{*}\right)=0$. From

$$
\hat{F}^{\prime}\left(x_{*}\right)=F^{\prime}\left(x_{*}\right)\left[I-A\left(A^{T} A\right)^{-1} A^{T}\right]
$$

and

$$
\nabla^{2} \hat{f}\left(x_{*}\right)=\hat{F}^{\prime}\left(x_{*}\right)^{T} \hat{F}^{\prime}\left(x_{*}\right)+\sum_{i=1}^{m} f_{i}\left(x_{*}\right) \nabla^{2} f_{i}\left(x_{*}\right)=\hat{F}^{\prime}\left(x_{*}\right)^{T} \hat{F}^{\prime}\left(x_{*}\right),
$$

we know that $\bar{\nabla}^{2} \hat{f}\left(x_{*}\right)$ has rank $n-k$.

By using (7.2) and (7.3), we created two sets of singular problems, with $\nabla^{2} \hat{f}\left(x_{*}\right)$ having rank $n-1$ and $n-2$, respectively, by using

$$
A \in \Re^{n \times 1}, \quad A^{T}=(1,0, \ldots, 0),
$$

and

$$
A \in \Re^{n \times 2}, \quad A^{T}=\left[\begin{array}{cccccccc}
1 & 0 & 0 & 0 & . & . & . & 0 \\
0 & 1 & 0 & 0 & . & . & . & 0
\end{array}\right],
$$

respectively. The reason for choosing unit vectors as columns for the matrix $A$ is mainly to preserve the sparsity of the Hessian during the transformation (7.2).

For all our test problems we used a standard line search backtracking strategy. All the test problems with the exception of rank $n-1$ and rank $n-2$ problems were run with analytic gradients and Hessians provided by the CUTE and MINPACK-2 collections. For rank $n-1$ and $n-2$ test problems, we have modified the analytic gradients provided by the CUTE collection to take into account the modification (7.2). On the other hand, we used the graph coloring algorithm [8] to evaluate the finite difference approximation of the Hessian matrix.

A summary for the test problems whose Hessians at the solution have ranks $n, n-1$, and $n-2$ is presented in Table 1 . The descriptions of the test problems and the detailed results are given in the Appendix. In Table 1 columns "better" and "worse" represent the number of times the tensor method was better and worse, respectively, than Newton's method by more than one gradient evaluation. The "tie" column represents the number of times the tensor and standard methods required within one gradient evaluation of each other. For each set of problems, we summarize the comparative costs of the tensor and standard methods using average ratios of three measures: gradient evaluations, function evaluations, and execution times. The average gradient evaluation ratio (geval) is the total number of gradients evaluations required by the tensor method, divided by the total number of gradients evaluations required by the standard method on these problems. The same measure is used for the average function evaluation (feval) and execution time (time) ratios. These average ratios include only problems that were successfully solved by both methods. We have excluded all cases where the tensor and standard methods converged to a different minimizer. However, the statistics for the "better,"- "worse," and "tie" columns include the cases where only one of the two methods converges, and exclude the cases where both methods do not converge. We also excluded problems requiring a number of gradient evaluations less or equal than 3 by both methods. Finally, columns " $\mathrm{t} / \mathrm{s}$ " and " $\mathrm{s} / \mathrm{t}$ " 
show the number of problems solved by the tensor method but not by the standard method and the number of problems solved by the standard method but not by the tensor method, respectively.

The improvement by the tensor method over the standard method on problems with rank $n-1$ is dramatic, averaging $48 \%$ in function evaluations, $52 \%$ in gradient evaluations, and $59 \%$ in execution times. This is due in part to the rate of convergence of the tensor method being faster than that of Newton's method, which is known to be only linearly convergent with constant $\frac{2}{3}$. On problems with rank $n-2$, the improvement by the tensor method over the standard method is also substantial, averaging $30 \%$ in function evaluations, $37 \%$ in gradient evaluations, and $34 \%$ in execution times. In the test results obtained for the nonsingular problems, the tensor method is $9 \%$ worse than the standard method in function evaluations, but $31 \%$ and $33 \%$ better in gradient evaluations and in execution times, respectively. The main reason for the tensor method requiring on the average more function evaluations than the standard method is because on some problems, the full tensor step does not provide sufficient decrease in the objective function, and therefore the tensor method has to perform a line search in both the Newton and tensor directions, which causes the number of function evaluations required by the tensor method to be inflated. As a result, we intend to investigate other possible global frameworks for line search methods that could potentially reduce the number of functions evaluations for the tensor method.

To obtain an experimental indication of the local convergence behavior of the tensor and Newton methods on problems where $\operatorname{rank}\left(\nabla^{2} f\left(x_{*}\right)\right)=n-1$, we examined the sequence of ratios

$$
\frac{\left\|x_{k}-x_{*}\right\|}{\left\|x_{k-1}-x_{*}\right\|}
$$

produced by the Newton and tensor methods on such problems. These ratios for a typical problem are given in Table 2. In almost all cases the standard method exhibits local linear convergence with constant near $\frac{2}{3}$, which is consistent with the theoretical analysis. The local convergence rate of the tensor method is faster, with a typical final ratio of around 0.01 . Whether this is a superlinear convergence remains to be determined. We have done similar experiments for problems with rank $\left(\nabla^{2} f\left(x_{*}\right)\right)=n-2$, and the tensor method did not show a faster-than-linear convergence rate, because it did not have enough information since $p=1$.

The tensor method solved a total of four nonsingular problems, five rank $n-1$ problems, and 7 rank $n-2$ problems that Newton's method failed to solve. The reverse never occurred. These results clearly indicate that the tensor method is most likely to be more robust than Newton's method.

The overall results show that having some extra information about the function and gradient. in the past step direction is quite useful in achieving the advantages of tensor methods.

\section{Summary and Future Research}

In this paper we presented new algorithms for solving large, sparse unconstrained optimization using tensor methods. Implementations using these tensor methods have been shown to be considerably more efficient especially on problems where the Hessian matrix has a small rank deficiency at the solution. Typical gains over standard Newton methods range from $40 \%$ to 
Table 1: Summary of the CUTE and MINPACK-2 test problems using line search

\begin{tabular}{|c|c|c|c|c|c|c|c|c|}
\hline Rank & \multicolumn{2}{|c|}{ Tensor/Standard } & \multicolumn{2}{c|}{ Pbs Solved } & \multicolumn{2}{c|}{ Average Ratio-Tensor/Standard } \\
\hline$\nabla^{2} f\left(x_{*}\right)$ & better & tie & worse & t/s & s/t & feval & geval & time \\
\hline$n$ & 53 & 38 & 5 & 4 & 0 & 1.09 & 0.69 & 0.67 \\
$n-1$ & 18 & 2 & 0 & 5 & 0 & 0.52 & 0.48 & 0.41 \\
$n-2$ & 18 & 1 & 1 & 7 & 0 & 0.70 & 0.63 & 0.66 \\
\hline
\end{tabular}

Table 2: Speed of convergence on the BRYBND problem with $\operatorname{rank}\left(\nabla^{2} f\left(x_{*}\right)\right)=n-1$, as modified by $(7.2), n=5000$, started from $x_{0}$. The ratios in second and third columns are defined by (7.4).

\begin{tabular}{|c|c|c|}
\hline Iteration $(k)$ & Standard Method & Tensor Method \\
\hline 1 & 0.659 & 0.659 \\
2 & 0.655 & 0.033 \\
3 & 0.650 & 0.459 \\
4 & 0.641 & 0.961 \\
5 & 0.629 & 0.850 \\
6 & 0.612 & 0.667 \\
7 & 0.590 & 0.410 \\
8 & 0.571 & 0.323 \\
9 & 0.600 & 0.126 \\
10 & 0.760 & 0.012 \\
11 & 0.940 & \\
12 & 0.988 & \\
13 & 0.970 & \\
14 & 0.969 & \\
15 & 0.956 & \\
16 & 0.926 & \\
17 & 0.891 & \\
18 & 0.909 & \\
19 & 0.848 & \\
20 & 0.926 & \\
21 & 0.939 & \\
22 & 0.896 & \\
23 & 0.832 & \\
24 & 0.871 & \\
25 & 0.742 & \\
26 & 0.667 & \\
27 & 0.667 & \\
28 & 0.666 & \\
29 & 0.665 & \\
30 & 0.666 & \\
& & \\
& & \\
\hline
\end{tabular}


$50 \%$ in function and gradient evaluations and in computer time. The size and consistency of the efficiency gains indicate that the tensor method may be preferable to Newton's method for solving large, sparse unconstrained optimization problems where analytic gradients and/or Hessians are available. To firmly establish such a conclusion, additional testing is required, including test problems of very large size.

On sparse problems where the function or the gradient is expensive to evaluate, the finite difference approximation of the Hessian matrix by the graph coloring algorithm [8] may be very costly. Hence, quasi-Newton methods may be preferable to use in this case. These methods involve low-rank corrections to a current approximate Hessian matrix. We are currently attempting to extend our tensor methods to quasi-Newton methods for large, sparse unconstrained minimization problems.

We also considered solving large, sparse, structured unconstrained optimization problems using tensor methods. In this variant, we explored the possibility of using exact third- and fourth-order derivative information. The calculation of these derivatives is simplified using the concept of partial separability, a structure that has already proven to be useful when building quadratic models for large-scale nonlinear problems [15]. The calculation of the minimizer of this exact tensor model is more problematic, however, because we need to solve a sparse system of nonlinear equations. An obvious approach to solve these equations is to use a Newton-like method. Such a method is characterized by the approximation of the Jacobian used in the Newton process. A simple idea is to use a fixed Jacobian at each step. This has the advantage that the Jacobian will have already been obtained in the current tensor iteration. However, potential slow convergence of such a scheme may make the cost of a tensor iteration prohibitive. We are currently investigating other possible approaches, such as a modified Newton's method in which the approximated Jacobian matrix will incorporate more useful information, or an iterative method such as a nonlinear GMRES. This work, a cooperation with Nick Gould [5], will be reported in the near future.

We are almost done with the implementation and testing of the two-dimensional trust region global strategy described in $\$ 5$. This work will be reported in a forthcoming paper.

We are also implementing the algorithms discussed in this paper in a software package. This package uses one past point in the formation of the tensor terms, which makes the additional cost and storage of the tensor method over the standard method very small. The package will be available soon.

Acknowledgments. We thank Professor Bobby Schnabel for his suggestions on how to minimize the tensor model when the Hessian is rank deficient, Nick Gould for discussing a number of implementation issues, Ta-Tung Chow for reviewing the first draft of the paper, and my CERFACS colleague Jacko Koster for his numerous suggestions. We also thank (rail Pieper from the MCS division at Argonne National Laboratory for her suggestions for improvement. 


\section{References}

[1] Anon. Harwell subroutine library (Release 11). Theoretical Studies Department, AEA Industrial Technology, 1993.

[2] B. M. Averick, R. G. Carter, J. J. Moré, and G. L. Xue. The MINPACK-2 test problem collection. Technical Report ANL/MCS-P153-0692, Argonne National Laboratory, Argonne, USA, 1992.

[3] I. Bongartz, A. R. Conn, N. I. M. Gould, and Ph. L. Toint. CUTE: Constrained and Unconstrained Testing Environment. Technical Report TR/PA/93/10, Centre Européen de Recherche et de Formation Avancée en Calcul Scientifique (CERFACS), Toulouse, France, 1993.

[4] A. Bouaricha. Solving large sparse systems of nonlinear equations and nonlinear least squares problems using tensor methods on sequential and parallel computers. Ph.D. thesis, Computer Science Department, University of Colorado at Boulder, 1992.

[5] A. Bouaricha and N. I. M. Gould. Personal communication. Centre Européen de Recherche et de Formation A vancée en Calcul Scientifique (CERFACS), Toulouse, France, 1994.

[6] A. Bouaricha and R. B. Schnabel. TENSOLVE: a software package for solving systems of nonlinear equations and nonlinear least squares problems using tensor methods. Technical Report TR/PA/93/23, Centre Européen de Recherche et de Formation Avancée en Calcul Scientifique (CERFACS), Toulouse, France, 1993.

[7] R. H. Byrd, R. B. Schnabel, and G. A. Shultz. Approximation solution of the trust region problem by minimization over two-dimensional subspaces. Math. Programming, 40:247-263, 1988.

[8] T. F. Coleman, B. S. Garbow, and J. J. Moré. Estimating sparse Hessian matrices. ACM Trans. Math. Software, 11:363-377, 1985.

[9] J. E. Dennis and R. B. Schnabel. Numerical methods for unconstrained optimization and nonlinear equations. Prentice-Hall, Englewood Cliffs, N.J., 1983.

[10] I. S. Duff and J. K. Reid. MA27: A set of Fortran subroutines for solving sparse symmetric sets of linear equations. Technical Report R-10533, AERE Harwell Laboratory, Harwell, UK, 1983.

[11] R. Fletcher. Practical method of optimization, volume 1, Unconstrained Optimization. John Wiley and Sons, New York, 1980.

[12] P. E. Gill, W. Murray, D. B. Ponceleon, and M. A. Saunders. Preconditioners for indefinite systems arising in optimization and nonlinear least squares problems. Technical Report SOL 90-x, Department of Operations Research, Stanford University, California, 1990.

[1:3] P. E. Gill, W. Murray, and M. H. Wright. Practical Optimization. Academic Press, London, 1981 . 
[14] A. Giriewank and M. R. Osborne. Analysis of Newton's method at irregular singularities. SIAM J. Numer. Anal., 18:145-150, 1981.

[15] A. Griewank and $\mathrm{Ph}$. L. Toint. On the unconstrained optimization of partially separable functions. In M. J. D. Powell, editor, Nonlinear Optimization 1981, pages 301-312. Academic Press, New York, 1982.

[16] J. J. Moré. The Levenberg-Marquardt algorithm: Implementation and theory. In G. A. Watson, editor, Proceedings Dundee 1977, Lecture Notes in Mathematics 630, pages 105116. Springer Verlag, Berlin, 1978.

[17] M. J. D. Powell. A new algorithm for unconstrained optimization. In J. B. Rosen, O. L. Mangasarian, and K. Ritter, editors, Nonlinear Programming, pages 33-65. Academic Press, New York, 1970.

[18] R. B. Schnabel and T. Chow. Tensor methods for unconstrained optimization using second derivatives. SIAM J. Optimization, 1:293-315, 1991.

[19] R. B. Schnabel and P. D. Frank. Tensor methods for nonlinear equations. SIAM J. Numer. Anal., 21:815-843; 1984 . 


\section{Appendix: Test Problems and Detailed Experimental Results}

The columns in Tables A-3-A-6 have the following meanings:

- func: name of the problem.

- $n$ : dimension of the problem.

- $x_{0}$ : starting point. $1,10,100$ stand for $x_{0}, 10 x_{0}$, and $100 x_{0}$, respectively.

- initf: initial value of the objective function.

- fcn: number of function evaluations.

- grad: number of gradient evaluations.

- time: execution time in seconds.

- final $f$ : final value of the objective function.

IL, NC stand for iteration limit exceeded and convergence to a nonminimizer, respectively. The iteration limit is 300 for the MINPACK-2 collection and 200 for the CUTE collection. All starting points were provided by the MINPACK-2 and CUTE collections.

Remark: For rank $n-1$ and $n-2$ problems grad does not include the number of gradients required by Hessian evaluations. On the other hand, $f c n$ does include the functions evaluations required by Hessian evaluations.

Table A-1: MINPACK-2 test problems

\begin{tabular}{|l|l|}
\hline Name & Description \\
\hline DEPT & Elastic-plastic torsion problem \\
DGL1 & Ginzburg-Landau (1-dimensional) superconductivity problem \\
DGL2 & Ginzburg-Landau (2-dimensional) superconductivity problem \\
DLJ2 & 2-dimensional Leonard-Jones clusters (molecular conformation) problem \\
DLJ3 & 3-dimensional Leonard-Jones clusters (molecular conformation) problem \\
DMSA & . Minimal surface area problem \\
DODC: & Optimal design with composite materials problem \\
DPJB & Pressure distribution in a journal bearing problem \\
DSSC: & Steady state combustion problem \\
& \\
\hline
\end{tabular}


Table A-2: CUTE test problems

\begin{tabular}{|c|c|}
\hline Name & Description \\
\hline ARWHEAD & $\begin{array}{l}\text { Quartic problem whose Hessian is an arrow-head (downwards) } \\
\text { with diagonal central part and border-width } 1\end{array}$ \\
\hline BDQRTIC & Quartic problem whose Hessian is banded with bandwidth 9 \\
\hline BRYBND & $\begin{array}{l}\text { Broyden banded system of nonlinear equations, considered in } \\
\text { the least square sense }\end{array}$ \\
\hline DIXMAANA & Dixon-Maany test problem (version A) \\
\hline DIXMAANB & Dixon-Maany test problem (version B) \\
\hline DIXMAANC & Dixon-Maany test problem (version $\mathrm{C}$ ) \\
\hline DIXMAANI & Dixon-Maany test problem (version I) \\
\hline DIXMAANJ & Dixon-Maany test problem (version $\mathrm{J}$ ) \\
\hline DIXON3DQ & Dixon's tridiagonal quadratic \\
\hline EDENSCH & Extended Dennis and Schnabel problem, as defined by $\mathrm{Li}$ \\
\hline ENGVAL1 & A sum of $2 n-2$ groups, $n-1$ of which contain 2 nonlinear elements \\
\hline FLETCBV2 & Boundary Value problem \\
\hline FREUROTH & Freudenstein and Roth test problem \\
\hline LIARWHD & A simplified version of the NONDIA problem \\
\hline MOREBV & $\begin{array}{l}\text { Boundary Value problem. This is the nonlinear least-squares } \\
\text { version without fixed variables }\end{array}$ \\
\hline NONDIA & Shanno's nondiagonal extension of Rosenbrock function \\
\hline NONDQUAR & $\begin{array}{l}\text { A nondiagonal quartic test problem with an } \\
\text { arrow-head type Hessian having a tridiagonal central part and } \\
\text { a border-width } 1 \text {. The Hessian is singular at the solution }\end{array}$ \\
\hline PENALTY 1 & $\begin{array}{l}\text { A sum of } n+1 \text { least-squares groups, the first } n \\
\text { which have only one linear element }\end{array}$ \\
\hline PENALTY2 & $\begin{array}{l}\text { A nonlinear least-squares problem with } m=2 n \text { groups, } \\
\text { group } 1 \text { is linear, groups } 2 \text { to } n \text { use } 2 \text { nonlinear elements, } \\
\text { groups } n+1 \text { to } m-1 \text { use } 1 \text { nonlinear element, and group } m \\
\text { uses } n \text { nonlinear elements }\end{array}$ \\
\hline POWELLSG; & Extended Powell singular problem \\
\hline QUARTC & A simple quartic function \\
\hline SINQUAD & A function with nontrivial groups and repetitious elements \\
\hline SROSENBR & Separable extension of Rosenbrock's function \\
\hline TQUARTIC: & A quartic function with nontrivial groups and repetitious elements \\
\hline TRIDLA & Shanno's TRIDIA quadratic tridiagonal problem \\
\hline WOODS & Extended Woods problem \\
\hline WOODS1 & Scaled extended Woods problem \\
\hline
\end{tabular}


Table A-3: Results of the MINPACK-2 test problems

\begin{tabular}{|c|c|c|c|c|c|c|c|c|c|c|c|}
\hline \multirow[b]{2}{*}{$f$ unc } & \multirow[b]{2}{*}{$n$} & \multirow[b]{2}{*}{$x_{0}$} & \multirow[b]{2}{*}{ instf } & \multicolumn{4}{|c|}{ Standard } & \multicolumn{4}{|c|}{ Tensor } \\
\hline & & & & Ich & grad & finalf & lime & $f c n$ & grad & finalf & time \\
\hline DEPT & 100 & 1 & $-0.36364 D+01$ & 2 & 2 & $-0.10694 D+02$ & $0.410 D .01$ & 2 & 2 & $.0 .10694 \mathrm{D}+02$ & 0.3910 .01 \\
\hline DEPT & 400 & $i$ & $-0.36584 \mathrm{D}+01$ & 2 & 2 & $-0.10902 \mathrm{D}+02$ & $0.180 D+00$ & 2 & 2 & $.0 .10902 \mathrm{D}+02$ & $0.182 D+00$ \\
\hline DEPT & 900 & 1 & $-0.36629 \mathrm{D}+01$ & 2 & 2 & $-0.10946 \mathrm{D}+02$ & $0.449 D+00$ & 2 & 2 & $.0 .10946 D+02$ & $0.471 D+00$ \\
\hline DEPT & 1600 & 1 & $-0.36645 \mathrm{D}+01$ & 2 & 2 & $-0.10961 \mathrm{D}+02$ & $0.900 \mathrm{D}+00$ & 2 & 2 & $.0 .10961 D+02$ & $0.900 \mathrm{D}+00$ \\
\hline DEPT & 2500 & 1 & $-0.36653 \mathrm{D}+01$ & 2 & 2 & $-0.10969 \mathrm{D}+02$ & $0.153 \mathrm{D}+01$ & 2 & 2 & $-0.10969 D+02$ & $0.151 D+01$ \\
\hline DEPT & 3600 & 1 & $-0.36657 \mathrm{D}+01$ & 2 & 2 & $.0 .10973 D+02$ & $0.239 D+01$ & 2 & 2 & $-0.10973 D+02$ & $0.236 \mathrm{D}+01$ \\
\hline DEPT & 4900 & 1 & $-0.366 .59 \mathrm{D}+01$ & 2 & 2 & $-0.10976 \mathrm{D}+02$ & $0.348 D+01$ & 2 & 2 & $.0 .10976 \mathrm{D}+02$ & $0.3490+01$ \\
\hline DEPT & 6400 & 1 & $-0.36661 \mathrm{D}+01$ & 2 & 2 & $-0.10977 D+02$ & $0.478 D+01$ & 2 & 2 & $-0.10977 D+02$ & $0.483 D+01$ \\
\hline DEPT & 8100 & 1 & $-0.36662 \mathrm{D}+01$ & 2 & 2 & $-0.10978 D+02$ & $0.746 \mathrm{D}+01$ & 2 & 2 & $-0.10978 D+02$ & $0.713 D+01$ \\
\hline DEPT & 10000 & 1 & $-0.36663 D+01$ & 2 & 2 & $.0 .10979 D+02$ & $0.833 D+01$ & 2 & 2 & $-0.10979 D+02$ & $0.831 D+01$ \\
\hline$D G L_{1}$ & 100 & 4 & $-0.16619 \mathrm{D}-03$ & 18 & 18 & $.0 .84562 D+04$ & $0.410 D+00$ & 5 & 5 & $-0.84562 \mathrm{D}+04$ & $0.1100+00$ \\
\hline DGLI & 400 & $i$ & $-0.16619 D-03$ & 18 & 18 & $-0.84562 D+04$ & $0.173 \mathrm{D}+01$ & 9 & 6 & $-0.84562 D+04$ & $0.620 D+00$ \\
\hline DGLI & 900 & 1 & $-0.16619 \mathrm{D}-03$ & 18 & 18 & $-0.84562 \mathrm{D}+04$ & $0.397 D+01$ & 6 & 6 & $-0.84562 D+04$ & $0.129 D+01$ \\
\hline DGLI & 1600 & 1 & $.0 .16619 D .03$ & 18 & 18 & $-0.84562 D+04$ & $0.706 D+01$ & 7 & 7 & $-0.84562 \mathrm{D}+04$ & $0.282 D+01$ \\
\hline DGLi & 2500 & $i$ & $.0 .16619 \mathrm{D} .03$ & 18 & 18 & $-0.84562 \mathrm{D}+04$ & $0.110 \mathrm{D}+02$ & 8 & 8 & $-0.845620+04$ & $0.512 D+01$ \\
\hline DGL1 & 3600 & 1 & $-0.16619 \mathrm{D}-03$ & 19 & 19 & $.0 .84562 \mathrm{D}+04$ & $0.169 D+02$ & 9 & 9 & $-0.84562 \mathrm{D}+04$ & $0.847 D+01$ \\
\hline DGL & 4900 & 1 & $-0.16619 \mathrm{D}-03$ & 19 & 19 & $-0.84562 D+04$ & $0.230 \mathrm{D}+02$ & 7 & 7 & $-0.84562 \mathrm{D}+04$ & $0.860 D+01$ \\
\hline $\mathrm{DGL}_{1}$ & 6400 & 1 & $.0 .16619 \mathrm{D}-03$ & 17 & 17 & $.0 .84+13 D+04$ & $0.270 D+02$ & 7 & 7 & $-0.84562 D+04$ & $0.115 \mathrm{D}+02$ \\
\hline DGLI & 8100 & 1 & $.0 .16619 \mathrm{D}-03$ & - & NC & - & - & 7 & 7 & $-0.84562 D+04$ & $0.149 D+02$ \\
\hline DGL1 & 10000 & 1 & $-0.16619 \mathrm{D}-03$ & - & NC & - & - & 9 & 3 & $-0.84562 \mathrm{D}+04$ & $0.236 \mathrm{D}+02$ \\
\hline DGL2 & 100 & 1 & $0.18190 \mathrm{D}+02$ & 231 & 84 & $0.16228 \mathrm{D}+02$ & $0.113 \mathrm{D}+02$ & 150 & 38 & $0.16228 \mathrm{D}+02$ & $0.531 D+01$ \\
\hline DGL2 & 400 & $\mathrm{t}$ & $0.201310+02$ & 159 & 67 & $0.162310+02$ & $0.450 \mathrm{D}+02$ & 210 & 43 & $0.162310+02$ & $0.307 \mathrm{D}+02$ \\
\hline DGL2 & 900 & $i$ & $0.22015 \mathrm{D}+02$ & 26.5 & 96 & $0.16232 \mathrm{D}+02$ & $0.202 \mathrm{D}+03$ & 418 & 76 & $0.16232 \mathrm{D}+02$ & $0.169 \mathrm{D}+03$ \\
\hline DGL2 & 1600 & 1 & $0.23884 D+02$ & 306 & 111 & $0.16232 \mathrm{D}+02$ & $0.584 D+03$ & 455 & 81 & $0.16232 \mathrm{D}+02$ & $0.444 \mathrm{D}+03$ \\
\hline DGL2 & 2500 & ! & $0.25748 D+02$ & 354 & 122 & $0.16232 D+02$ & $0.133 D+04$ & 607 & 102 & $0.16232 \mathrm{D}+02$ & $0.117 D+04$ \\
\hline DGL2 & 3600 & 1 & $0.27609 \mathrm{D}+02$ & 503 & 165 & $0.16232 \mathrm{D}+02$ & $0.314 D+04$ & 7.51 & 137 & $0.16232 \mathrm{D}+02$ & $0.219 D+04$ \\
\hline DGL2 & 4900 & 1 & $0.29469 D+02$ & 686 & 223 & $0.16232 \mathrm{D}+02$ & $0.128 D+0.5$ & 849 & 144 & $0.16232 \mathrm{D}+02$ & $0.644 \mathrm{D}+04$ \\
\hline$D L J 2$ & 100 & 1 & $-0.10698 D+03$ & 252 & 107 & $.0 .13375 D+03$ & $0.113 D+03$ & 176 & 51 & $=0.13396 \mathrm{D}+03$ & $0.544 \mathrm{D}+02$ \\
\hline DLJ2 & 200 & 1 & $-0.22945 \mathrm{D}+03$ & 105 & 132 & $-0.28056 D+03$ & $0.1030+04$ & 475 & 89 & $-0.281400+03$ & $0.698 D+03$ \\
\hline$D L J 2$ & 300 & 1 & $-0.352610+03$ & 544 & 145 & $.0 .14216 D+03$ & $0.372 D+04$ & 631 & 118 & $-0.44025 D+03$ & $0.305 \mathrm{D}+04$ \\
\hline DLJ3 & 120 & 1 & $.0 .11782 D+03$ & 375 & 112 & $.0 .17954 D+03$ & $0.137 \mathrm{D}+03$ & 348 & 65 & $-0.17073 D+03$ & $0.805 \mathrm{D}+02$ \\
\hline DLJ3 & 210 & 1. & $-0.23253 D+03$ & 485 & 139 & $-0.34073 D+03$ & $0.838 D+03$ & 608 & 113 & $-0.34522 \mathrm{D}+03$ & $0.687 D+03$ \\
\hline$D L \sqrt{3}$ & 360 & 1. & $.0 .42908 D+03$ & $103 !$ & 281 & $-0.6374+D+03$ & $0.826 \mathrm{D}+04$ & 963 & 173 & $-0.63311 \mathrm{D}+03$ & $0.466 \mathrm{D}+04$ \\
\hline$D M S A$ & 100 & $i$ & $0.14608 D+01$ & 4 & 4 & $0.14185 \mathrm{D}+01$ & $0.1500+00$ & 4 & 4 & $0.14185 \mathrm{D}+01$ & $0.160 \mathrm{D}+00$ \\
\hline$D M S A$ & 400 & 1 & $0.14891 D+01$ & 4 & 4 & $0.142060+01$ & $0.640 \mathrm{D}+00$ & 10 & 4 & $0.14206 D+01$ & $0.7100+00$ \\
\hline DMSA & 900 & 1 & $0.150350+01$ & $s$ & 5 & $0.14210 D+01$ & $0.212 D+01$ & 4 & 4 & $0.142100+01$ & $0.1720+01$ \\
\hline DMSA & 1600 & $i$ & $0.15123 \mathrm{D}+01$ & -.5 & 5 & $0.14212 D+01$ & $0.396 \mathrm{D}+0 \mathrm{i}$ & 10 & 5 & $0.14212 \mathrm{D}+01$ & $0.446 D+01$ \\
\hline DMSA & 2500 & 1 & $0.15183 D+01$ & 6 & 6 & $0.14212 D+01$ & $0.833 D+01$ & 14 & 5 & $0.14212 \mathrm{D}+01$ & $0.7610+01$ \\
\hline DMSA & 3600 & $i$ & $0.15227 D+01$ & 6 & 6 & $0.14213 D+01$ & $0.130 D+02$ & 10 & 6 & $0.142130+01$ & $0.146 \mathrm{D}+02$ \\
\hline DMSA & 4900 & 1 & $0.15260 D+01$ & 6 & 6 & $0.14213 D+01$ & $0.190 D+02$ & 11 & 6 & $0.14213 D+01$ & $0.2100+02$ \\
\hline DMSA & 6400 & 1 & $0.15286 \mathrm{D}+01$ & 7 & 7 & $0.142130+01$ & $0.308 D+02$ & 9 & 7 & $0.14213 D+01$ & $0.342 \mathrm{D}+02$ \\
\hline$D M S A$ & 8100 & 1 & $0.15307 D+01$ & 17 & 12 & $0.142130+01$ & $0.846 D+02$ & 16 & 8 & $0.14213 D+01$ & $0.595 \mathrm{D}+02$ \\
\hline DMSA & 10000 & 1 & $0.153240+01$ & 21. & 14 & $0.142130+01$ & $0.117 D+03$ & 17 & $T$ & $0.142130+01$ & $0.6010+02$ \\
\hline DODC. & 100 & 1 & $0.446260-01$ & 14 & 8 & $-0.10980 D-01$ & $0.420 D+00$ & 16 & 8 & $-0.109800-01$ & $0.487 D+00$ \\
\hline$D, D C$ & 400 & 1 & $0.47194 \mathrm{D}-01$ & 13 & 10 & .0 .112480 .01 & $0.234 \mathrm{D}+01$ & 19 & 10 & $-0.11248 D .01$ & $0.272 \mathrm{D}+01$ \\
\hline DODC & 300 & 1 & 0.477710 .01 & 23 & 13 & .0 .113290 .01 & $0.744 D+01$ & 41 & 14 & $-0.113290-01$ & $0.943 D+01$ \\
\hline DUDC & 1600 & l & 0.479740 .01 & 5.5 & 23 & $=0.11351 \mathrm{D}-01$ & $0.256 \mathrm{D}+02$ & $56=$ & 21 & $-0.11351 \mathrm{D}-01$ & $0.2670+02$ \\
\hline DUDC & 2.500 & 1 & $0.48082 \mathrm{D} .01$ & 70 & .33 & $-0.11359 \mathrm{D}-01$ & $0.617 \mathrm{D}+02$ & 117 & 28 & $-0.11 .3590-01$ & $0.623 \mathrm{D}+02$ \\
\hline DODC & 3600 & 1 & $0.481 .39 \mathrm{D}-01$ & 129 & 49 & $-0.11368 \mathrm{D}-01$ & $0.148 D+03$ & 194 & 42 & $-0.11368 \mathrm{D}-01$ & $0.144 D+0.3$ \\
\hline DUDC. & 4900 & $i$ & $0.48178 \mathrm{D}-01$ & 565 & 163 & .0 .113720 .01 & $0.313 D+0.3$ & 406 & 76 & -0.113720 .01 & $0.3800+03$ \\
\hline$D O D C$ & 6400 & I & $0.18202 \mathrm{D} .01$ & 597 & 168 & $-0.11 .374 D .01$ & $0.999 D+03$ & 526 & 94 & $.0 .11374 D .01$ & $0.640 D+03$ \\
\hline DUDC & 8100 & $i$ & $0.18221 \mathrm{D} .01$ & - & IL & - & - & - & it & - & - \\
\hline DODC & 10000 & $i$ & $0.18234 \mathrm{D} .01$ & - & IL & _- & - & - & IL & - & - \\
\hline DPJB & 100 & 1 & $0.11274 D+02$ & 2 & 2 & $-0.27881 D+00$ & $0.488 D .01$ & 2 & 2 & $-0.27881 D+00$ & $0.508 \mathrm{D}-01$ \\
\hline DPJB & 400 & 1 & $0.1333310+02$ & 2 & 2 & $.0 .28144 \mathrm{D}+00$ & $0.209 D+00$ & 2 & 2 & $-0.28144 D+00$ & $0.201 D+00$ \\
\hline DPJB & 300 & 1 & $0.14544 D+02$ & 2 & 2 & $-0.28213 \mathrm{D}+00$ & $0.500 \mathrm{D}+00$ & 2 & 2 & $-0.28219 D+00$ & $0.430 \mathrm{D}+00$ \\
\hline$D F \cdot 3 B$ & 1600 & $i$ & $0.155,45 \mathrm{D}+02$ & 2 & 2 & $-0.28249 D+00$ & $0.333 D+00$ & 2 & 2 & $-0.282490+00$ & $0.9590+00$ \\
\hline$D F \cdot J B$ & 2500 & i & $0.16462 \mathrm{D}+02$ & 2 & 2 & $.0 .28264 D+00$ & $0.150 D+01$ & 2 & 2 & $-0.282640+00$ & $0.160 \mathrm{D}+01$ \\
\hline DPJB & 3600 & 1 & $0.173360+02$ & 2 & 2 & $-0.28272 D+00$ & $0.24 .3 \mathrm{D}+01$ & 2 & 2 & $-0.28272 \mathrm{D}+00$ & $0.256 \mathrm{D}+01$ \\
\hline$D P J B$ & 4900 & 1 & $0.18186 \mathrm{D}+02$ & 2 & 2 & $-0.28277 D+00$ & $0.374 \mathrm{D}+01$ & 2 & 2 & $-0.232 \div 7 D+00$ & $0.362 \mathrm{D}+01$ \\
\hline$D F^{\prime} J B$ & 6400 & 1 & $0.19022 D+02$ & 2 & 2 & $-0.28280 D+00$ & $0.496 \mathrm{D}+01$ & 2 & 2 & $-0.28280 D+00$ & $0.489 D+01$ \\
\hline DPIB & 8100 & $i$ & $0.12848 D+02$ & 2 & 2 & $-0.28282 \mathrm{D}+00$ & $0.7330 \mathrm{D}+01$ & 2 & 2 & $-0.28282 D+00$ & $0.741 D+01$ \\
\hline DPJB & 10000 & 1. & $0.20666 \mathrm{D}+02$ & 2 & 2 & $.0 .28284 \mathrm{D}+00$ & $0.8780+01$ & 2 & 2 & $-0.28284 D+00$ & $0.862 D+01$ \\
\hline DSOC & 100 & 1 & $-0.52548 D+01$ & 3 & 3 & $-0.55973 D+01$ & $0.1100+00$ & 3 & 3 & $-0.55979 D+01$ & $0.1200+00$ \\
\hline 0.336 & 400 & $i$ & $-0.505070+01$ & 3 & 3 & $.0 .560770+01$ & $0.510 D+00$ & 3 & 3 & $-0.56077 \mathrm{D}+01$ & $0.540 D+00$ \\
\hline DSSC: & 300 & 1 & $-0.49189 D+01$ & 3 & 3 & $-0.56098 D+01$ & $0.1200+01$ & 3 & 3 & $-0.56038 D+01$ & $0.131 D+01$ \\
\hline 0.350 & 1600 & 1 & $-0.48224 D+01$ & 3 & 3 & $-0.56105 D+01$ & $0.223 D+01$ & 3 & 3 & $-0.56105 D+01$ & $0.246 \mathrm{D}+01$ \\
\hline DSSC & 2500 & $i$ & $-0.47466 D+01$ & .3 & 3 & $-0.56108 D+01$ & $0.382 \mathrm{D}+01$ & 3 & 3 & $-0.56108 \mathrm{D}+01$ & $0.413 D+01$ \\
\hline DisC: & .3600 & 1 & $-0.46842 D+01$ & 3 & 3 & $-0.56110 D+01$ & $0.53 .5 D+01$ & 3 & 3 & $.0 .56110 \mathrm{D}+01$ & $0.624 D+01$ \\
\hline DSSC: & 4900 & 1 & $-0.46312 D+01$ & .3 & 3 & $.0 .56112 \mathrm{D}+01$ & $0.880 D+01$ & 3 & 3 & $-0.56112 \mathrm{D}+01$ & $0.313 D+01$ \\
\hline DSsC: & 6400 & 1 & $.0 .458520+01$ & 3 & 3 & $.0 .561120+0 i$ & $0.1150+02$ & 3 & 3 & $-0.56112 D+0 \mathrm{i}$ & $0.122 \mathrm{D}+02$ \\
\hline DSSC & $\$ 100$ & 1 & $-0.45445 \mathrm{D}+01$ & 3 & 3 & $.0 .5611 .3 D+01$ & $0.173 \mathrm{D}+02$ & 3 & 3 & $-0.56113 D+01$ & $0.173 D+02$ \\
\hline 0.536 & 10000 & $i$ & $-0.45080 \mathrm{D}+01$ & 2 & 2 & $.0 .5611 .3 D+01$ & $0.102 D+02$ & 2 & 2 & $-0.56113 \mathrm{D}+01$ & $0.102 D+02$ \\
\hline
\end{tabular}


Table A-4: Results of the CUTE test problems

\begin{tabular}{|c|c|c|c|c|c|c|c|c|c|c|c|}
\hline \multirow[b]{2}{*}{ func. } & \multirow[b]{2}{*}{$n$} & \multirow[b]{2}{*}{$x_{0}$} & \multirow[b]{2}{*}{ initf } & \multicolumn{4}{|c|}{ Standard } & \multicolumn{4}{|c|}{ Teasor } \\
\hline & & & & fen & grad & $f$ inalf & time & fen & grad & finalf & time \\
\hline \multirow[t]{3}{*}{ ARWHEAD } & 5000 & 1 & $0.14997 D+0.5$ & 7 & 7 & $0.00000 D+00$ & $0.496 D+02$ & 3 & 3 & $0.00000 \mathrm{D}+00$ & $0.168 D+02$ \\
\hline & & 10 & $0.19978 D+09$ & 12 & 12 & $0.00000 \mathrm{D}+00$ & $0.909 D+02$ & 18 & 14 & $0.00000 \mathrm{D}+00$ & $0.1100+03$ \\
\hline & & 100 & $0.19996 \mathrm{D}+13$ & 18 & 18 & $0.000000+00$ & $0.140 D+03$ & 33 & 20 & $0.00000 \mathrm{D}+00$ & $0.160 D+0.3$ \\
\hline \multirow[t]{3}{*}{ BDQRTIC } & 1000 & 1 & $0.225100+06$ & 10 & 10 & $0.39838 D+04$ & $0.992 D+01$ & 24 & 12 & $0.39838 \mathrm{D}+04$ & $0.127 D+02$ \\
\hline & & 10 & $0.22424 D+10$ & 16 & 16 & $0.39838 D+04$ & $0.165 D+02$ & 38 & 17 & $0.39838 D+04$ & $0.185 D+02$ \\
\hline & & 100 & $0.22410 D+14$ & 22 & 22 & $0.39838 D+04$ & $0.231 D+02$ & 51 & 23 & $0.39838 \mathrm{D}+04$ & $0.254 \mathrm{D}+02$ \\
\hline \multirow[t]{3}{*}{ BRYBND } & .5000 & 1 & $0.12490 D+06$ & 24 & 17 & $0.13587 \mathrm{D} \cdot 19$ & $0.327 \mathrm{D}+02$ & 49 & 16 & $0.12928 \mathrm{D}-16$ & $0.381 D+02$ \\
\hline & & 10 & $0.10765 D+12$ & 37 & 26 & $0.14231 D-19$ & $0.510 D+02$ & 50 & 24 & $0.98532 \mathrm{D}-17$ & $0.551 \mathrm{D}+02$ \\
\hline & & 100 & $0.12303 D+18$ & - & IL & - & - & 810 & 189 & $0.35466 \mathrm{D}-16$ & $0.473 \mathrm{D}+03$ \\
\hline \multirow[t]{3}{*}{ DIXON3DQ } & 5000 & 1 & $0.80000 D+01$ & 2 & 2 & $0.11414 \mathrm{D} .24$ & $0.600 \mathrm{D}+00$ & 2 & 2 & $0.11414 \mathrm{D}-24$ & $0.560 \mathrm{D}+00$ \\
\hline & & 10 & $-0.24200 D+03$ & 2 & 2 & $0.34514 \mathrm{D}-23$ & $0.570 D+00$ & 2 & 2 & $0.34514 \mathrm{D}-23$ & $0.570 D+\infty 0$ \\
\hline & & 100 & $0.20402 D+05$ & 2 & 2 & $0.29050 \mathrm{D} .21$ & $0.560 D+00$ & 2 & 2 & $0.29050 \mathrm{D}-21$ & $0.560 D+00$ \\
\hline \multirow[t]{3}{*}{ DIXMAANA } & 3000 & 1 & $0.20501 \mathrm{D}+0.5$ & 6 & 6 & $0.10000 D+01$ & $0.165 \mathrm{D}+01$ & 8 & 6 & $0.10000 D+01$ & $0.205 \mathrm{D}+01$ \\
\hline & & 10 & $0.80013 D+10$ & 18 & 12 & $0.100000+01$ & $0.366 \mathrm{D}+0.2$ & 19 & 12 & $0.10000 D+01$ & $0.455 D+01$ \\
\hline & & 100 & $0.80000 D+16$ & 29 & 21 & $0.100000+01$ & $0.654 D+01$ & 19 & 19 & $0.10000 D+01$ & $0.724 D+01$ \\
\hline DIXMAANB & 3000 & 1 & $0.43242 D+05$ & 6 & 6 & $0.100000+01$ & $0.162 D+01$ & 15 & 6 & $0.100000+01$ & $0.218 D+01$ \\
\hline & & 10 & $0.17227 D+11$ & - & IL & - & - & - & IL & - & - \\
\hline & & 100 & $0.16116 D+17$ & - & IL & - & - & - & IL & - & - \\
\hline DIXMAANC. & 3000 & 1 & $0.74433 D+0.5$ & 15 & 15 & $0.10000 D+01$ & $0.450 \mathrm{D}+01$ & 1.5 & 13 & $0.10000 D+01$ & $0.506 \mathrm{D}+01$ \\
\hline & & 10 & $0.34452 D+11$ & - & IL & - & - & - & IL & - & - \\
\hline & & 100 & $0.322330+17$ & - & IL & - & - & - & IL & - & - \\
\hline DIXMAANI & 3000 & 1 & $0.12022 D+0.5$ & 100 & 33 & $0.10000 D+01$ & $0.119 D+02$ & 108 & 18 & $0.10000 D+01$ & $0.907 D+01$ \\
\hline & & 10 & $0.80004 D+10$ & 184 & 58 & $0.10000 D+01$ & $0.217 D+02$ & 152 & 32 & $0.10000 \mathrm{D}+01$ & $0.1570+02$ \\
\hline & & 100 & $0.80000 D+16$ & 263 & 77 & $0.10000 D+01$ & $0.287 D+02$ & 247 & 41 & $0.10000 D+01$ & $0.209 \mathrm{D}+02$ \\
\hline DIXMAANJ & 3000 & 1 & $0.00000 D+00$ & 1 & 1 & $0.000000+00$ & $0.900 \mathrm{D}-01$ & 1 & 1 & $0.00000 D+00$ & $0.7000-01$ \\
\hline & & 10 & $0.00000 D+00$ & 1 & 1 & $0.000000+00$ & $0.700 \mathrm{D}-01$ & 1 & $i$ & $0.00000 D+00$ & $0.700 D-01$ \\
\hline & & 100 & $0.000000+00$ & 1 & 1 & $0.00000 D+00$ & $0.700 \mathrm{D} .01$ & 1 & 1 & $0.00000 D+00$ & $0.700 D-01$ \\
\hline EDENSCE & 2000 & 1 & $0.73583 \mathrm{D}+07$ & 13 & - 13 & $0.12003 D+05$ & $0.442 D+01$ & 31 & 16 & $0.12003 \mathrm{D}+05$ & $0.666 \mathrm{D}+01$ \\
\hline & & 10 & $0.15 \div 84 D+12$ & 19 & 19 & $0.12003 D+05$ & $0.666 \mathrm{D}+0 \mathrm{I}$ & 53 & 20 & $0.12003 \mathrm{D}+05$ & $0.877 D+01$ \\
\hline & & 100 & $0.16253 \mathrm{D}+16$ & 24 & 24 & $0.12003 D+05$ & $0.848 D+01$ & 48 & 25 & $0.12003 D+0.5$ & $0.106 \mathrm{D}+02$ \\
\hline ENGVAL 1 & 3000 & 1 & $0.29494 D+06$ & 8 & 8 & $0.55487 \mathrm{D}+04$ & $0.536 \mathrm{D}+01$ & 7 & 7 & $0.55487 \mathrm{D}+04$ & $0.548 D+01$ \\
\hline & & 10 & $0.31990 D+10$ & 14 & 14 & $0.5 .5487 D+04$ & $0.983 D+01$ & 27 & 14 & $0.55487 \mathrm{D}+04$ & $0.124 D+02$ \\
\hline & & 100 & $0.31994 D+14$ & 20 & 20 & $0.53487 D+04$ & $0.143 D+02$ & 49 & 20 & $0.55487 D+04$ & $0.1860+02$ \\
\hline FLETCBV2 & 10000 & 1 & $-0.500130+00$ & 1 & 1 & $0.00000 D+00$ & $0.460 \mathrm{D}+00$ & 1 & 1 & $0.00000 D+00$ & $0.380 \mathrm{D}+00$ \\
\hline & & 10 & $0.39995 \mathrm{D}+02$ & 2 & 2 & $-0.50013 D+00$ & $0.2070+01$ & 2 & 2 & $-0.500130+00$ & $0.215 D+01$ \\
\hline & & 100 & $0.48995 D+04$ & 2 & 2 & $-0.50013 D+00$ & $0.212 D+01$ & 2 & 2 & $-0.50013 \mathrm{D}+00$ & $0.212 D+01$ \\
\hline FREUROTH & 5000 & 1 & $0.50486 \mathrm{D}+07$ & 461 & 83 & $0.60793 \mathrm{D}+06$ & $0.956 \mathrm{D}+02$ & 424 & 53 & $0.60821 D+06$ & $0.785 \mathrm{D}+02$ \\
\hline & & 10 & $0.15963 \mathrm{D}+09$ & 444 & 77 & $0.60726 \mathrm{D}+06$ & $0.894 \mathrm{D}+02$ & 200 & 30 & $0.35200 \mathrm{D}+07$ & $0.414 \mathrm{D}+02$ \\
\hline & & 100 & $0.13056 \mathrm{D}+15$ & 92 & 45 & $0.42206 D+06$ & $0.426 \mathrm{D}+02$ & 155 & 51 & $0.53488 D+06$ & $0.605 \mathrm{D}+02$ \\
\hline LIARWHD & 10000 & 1 & $0.58500 \mathrm{D}+07$ & 13 & 13 & $0.81983 \mathrm{D}-21$ & $0.217 D+03$ & 13 & 3 & $0.49397 \mathrm{D}-27$ & $0.148 D+03$ \\
\hline & & 10 & $0.07359 D+11$ & 22 & 21 & $0.63218 \mathrm{D}-17$ & $0.363 \mathrm{D}+0.3$ & 24 & 12 & $0.11125 \mathrm{D}-16$ & $0.205 D+03$ \\
\hline & & 100 & $0.101830+16$ & 26 & 26 & $0.16259 \mathrm{D}-16$ & $0.463 D+0.3=$ & 48 & 18 & $0.31712 \mathrm{D}-21$ & $0.319 D+03$ \\
\hline MOKEBV & 5000 & 1 & $0.15969 \mathrm{D} .06$ & 2 & 2 & $0.58271 D-14$ & $0.100 D+01$ & 2 & 2 & 0.58271 D. 14 & $0.9400+00$ \\
\hline & & 10 & $0.15983 \mathrm{D}-04$ & 2 & 2 & $0.22833 \mathrm{D}-09$ & $0.950 \mathrm{D}+00$ & 2 & 2 & 0.228330 .09 & $0.9600+00$ \\
\hline & & 100 & $0.17190 \mathrm{D}-02$ & 2 & 2 & $0.32151 \mathrm{D} .04$ & $0.910 D+00$ & 2 & 2 & $0.321510-04$ & $0.910 D+00$ \\
\hline NONDIA & 10000 & 1 & $0.39996 \mathrm{D}+07$ & 6 & 6 & $0.476 .32 \mathrm{D}-24$ & $0.909 D+02$ & 10 & 5 & $0.11200 D-20$ & $0.737 D+02$ \\
\hline & & 10 & $0.12099 D+11$ & 34 & 34 & $0.53482 \mathrm{D} .25$ & $0.595 \mathrm{D}+0.3$ & 20 & 16 & $0.19919 \mathrm{D}-28$ & $0.274 D+03$ \\
\hline & & 100 & $0.102000+15$ & 39 & 39 & $0.22 .382 \mathrm{D}-20$ & $0.6810+03$ & 52 & 21 & $0.6573 .3 \mathrm{D}-17$ & $0.367 D+0.3$ \\
\hline NINDQUAR & 10000 & 1 & $0.10006 D+05$ & 20 & 20 & $0.41398 \mathrm{D} .03$ & $0.3650+03$ & 20 & 20 & $0.414130-03$ & $0.9700+0.3$ \\
\hline & & 10 & $0.39981 D+08$ & 25 & 25 & $0.12450 \mathrm{D} .08$ & $0.122 \mathrm{D}+04$ & 25 & 25 & $0.125 .38 \mathrm{D}-08$ & $0.123 \mathrm{D}+04$ \\
\hline & & 100 & $0.39980 \mathrm{D}+12$ & 31 & 31 & $0.73954 \mathrm{Q}-09$ & $0.152 \mathrm{D}+04$ & 31 & 31 & 0.872100 .09 & $0.1530+04$ \\
\hline PENALTYI & 100 & 1 & $0.11448 D+12$ & 47 & 38 & $0.30255 \mathrm{D}-03$ & $0.493 \mathrm{D}+01$ & 10 & 7 & $0.90249 D-0.3$ & $0.7800+00$ \\
\hline & & 10 & $0.11448 D+16$ & 51 & 43 & $0.30255 \mathrm{D}-0.3$ & $0.557 \mathrm{D}+01$ & 7 & 7 & 0.902430 .0 .3 & $0.8500+00$ \\
\hline & & 100 & $0.11448 \mathrm{D}+20$ & 5.5 & 48 & $0.30257 D-0.3$ & $0.625 D+01$ & 30 & 16 & $0.30252 D-0.3$ & $0.213 D+01$ \\
\hline PENALTY 2 & 100 & 1 & $0.1688 .5 \mathrm{D}+07$ & 24 & 21 & $0.37096 \mathrm{D}+0.5$ & $0.296 \mathrm{D}+01$ & 26 & 20 & $0.97096 \mathrm{D}+0.5$ & $0.3000+01$ \\
\hline & & 10 & $0.15339 D+11$ & 27 & 26 & $0.37036 \mathrm{D}+05$ & $0.369 D+01$ & 47 & 27 & $0.37096 \mathrm{D}+0.5$ & $0.4110+01$ \\
\hline & & 100 & $0.159330 D+15$ & $3 !$ & 31 & $0.37096 \mathrm{D}+0.5$ & $0.444 D+01$ & 70 & 31 & $0.37096 D+0.5$ & $0.481 D+01$ \\
\hline POWELLSG & 10000 & 1 & $0.5 .3750 D+06$ & 16 & 16 & 0.109470 .04 & $0.143 D+02$ & 33 & 15 & $0.83906 \mathrm{D}-0.5$ & $0.179 D+02$ \\
\hline & & 10 & $0.40 .38 .5 D+10$ & 21 & 21 & $0.32320 \mathrm{D}-04$ & $0.130 \mathrm{D}+02$ & 28 & 22 & 0.1163 .50 .04 & $0.257 \mathrm{D}+02$ \\
\hline & & 100 & $0.40251 D+14$ & 27 & 27 & $0.19556 \mathrm{D}-04$ & $0.247 D+02$ & 31 & 27 & $0.540 .51 D-0.5$ & $0.316 D+02$ \\
\hline QUARTC & 1000 & 1 & $0.13850 \mathrm{D}+15$ & 3.5 & 35 & $0.22354 \mathrm{D} .09$ & $0.231 D+01$ & 35 & 35 & $0.223 .54 \mathrm{D} .09$ & $0.2870+01$ \\
\hline & & 10 & $0.18125 D+15$ & 35 & 35 & $0.20411 D-09$ & $0.229 D+01$ & 3.5 & 35 & 0.204110 .03 & $0.285 \mathrm{D}+0 \mathrm{~L}$ \\
\hline & & 100 & $0.65804 D+14$ & 34 & 34 & $0.37515 \mathrm{D}-09$ & $0.223 D+01$ & 35 & 34 & $0.37515 \mathrm{D}-03$ & $0.278 D+01$ \\
\hline
\end{tabular}


Table A-4: Results of the CUTE test problems (continued)

\begin{tabular}{|c|c|c|c|c|c|c|c|c|c|c|c|}
\hline \multirow[b]{2}{*}{ func } & \multirow[b]{2}{*}{$n$} & \multirow[b]{2}{*}{$x_{0}$} & \multirow[b]{2}{*}{ initf } & \multicolumn{4}{|c|}{ Standard } & \multicolumn{4}{|c|}{ Tensor } \\
\hline & & & & $f c n$ & grad & finolf & time & $f \mathrm{cn}$ & grad & finalf & time \\
\hline \multirow[t]{3}{*}{ SINQUAD } & 10000 & 1 & $0.65610 D+00$ & 25 & 20 & $0.39609 \mathrm{D}-10$ & $0.975 D+03$ & 66 & 21 & $0.35876 \mathrm{D}-15$ & $0.103 D+04$ \\
\hline & & 10 & $0.00000 D+00$ & 1 & 1 & $0.35876 \mathrm{D}-15$ & $0.290 D+00$ & 1 & 1 & $0.35876 \mathrm{D}-15$ & $0.300 \mathrm{D}+00$ \\
\hline & & 100 & $0.65610 D+04$ & 18 & 18 & $0.69625 \mathrm{D}-08$ & $0.881 D+03$ & 47 & 19 & $0.425240-15$ & $0.966 D+03$ \\
\hline \multirow[t]{3}{*}{ SRUSENBR } & 5000 & 1 & $0.48500 \mathrm{D}+0 \mathrm{~s}$ & 9 & 8 & $0.93253 \mathrm{D}-11$ & $0.297 D+01$ & 16 & 7 & 0.109270 .17 & $0.332 \mathrm{D}+01$ \\
\hline & & 10 & $0.44893 \mathrm{D}+10$ & 97 & 66 & $0.38588 D-18$ & $0.279 D+02$ & 65 & 33 & $0.22535 \mathrm{D} .15$ & $0.179 D+02$ \\
\hline & & 100 & $0.51123 D+14$ & - & IL & - & $=$ & 204 & 97 & $0.26051 \mathrm{D}-08$ & $0.547 \mathrm{D}+02$ \\
\hline \multirow[t]{3}{*}{ TQUARTIC } & 1000 & 1 & $0.81000 \mathrm{D}+00$ & 2 & 2 & $0.39936 \mathrm{D} .27$ & $0.270 \mathrm{D}+00$ & 2 & 2 & $0.39936 \mathrm{D}-27$ & $0.260 D+00$ \\
\hline & & 10 & $0.00000 D+00$ & 1 & $\mathbf{t}$ & $0.39936 \mathrm{D}-27$ & 0.2000 .01 & 1 & 1 & $0.39936 \mathrm{D}-27$ & $0.200 \mathrm{D}-01$ \\
\hline & & 100 & $0.81000 D+02$ & 2 & 2 & $0.12622 \mathrm{D}-24$ & $0.260 D+00$ & 2 & 2 & $0.12622 \mathrm{D}-24$ & $0.260 D+00$ \\
\hline \multirow[t]{3}{*}{ TRIDIA } & 10000 & 1 & $0.50005 \mathrm{D}+08$ & 2 & 2 & $0.41242 D-24$ & $0.119 \mathrm{D}+01$ & 2 & 2 & $0.41242 \mathrm{D} .24$ & $0.117 D+01$ \\
\hline & & 10 & $0.50005 \mathrm{D}+10$ & 2 & 2 & $0.13131 \mathrm{D}-22$ & $0.117 D+01$ & 2 & 2 & 0.131310 .22 & $0.117 \mathrm{D}+01$ \\
\hline & & 100 & $0.50005 D+12$ & 2 & 2 & $0.33835 \mathrm{D}-20$ & $0.1170+01$ & 2 & 2 & $0.33835 \mathrm{D}-20$ & $0.117 D+01$ \\
\hline \multirow[t]{3}{*}{ woODs } & 10000 & 1 & $0.27296 D+08$ & 28 & 23 & $0.31973 \mathrm{D} .14$ & $0.259 D+02$ & 49 & 21 & $0.33996 \mathrm{D}-17$ & $0.305 D+02$ \\
\hline & & 10 & $0.22566 \mathrm{D}+12$ & 51 & 42 & $0.42521 \mathrm{D}-12$ & $0.484 D+02$ & 72 & 34 & $0.42039 \mathrm{D}-09$ & $0.503 D+02$ \\
\hline & & 100 & $0.22122 \mathrm{D}+16$ & 73 & 60 & $0.27578 \mathrm{D}-10$ & $0.698 D+02$ & 100 & 49 & $0.16526 \mathrm{D}-16$ & $0.730 \mathrm{D}+02$ \\
\hline \multirow[t]{3}{*}{ WOODSI } & 10000 & 1 & $0.53500 \mathrm{D}+06$ & 9 & 9 & $0.17486 \mathrm{D} \cdot 11$ & $0.9490+01$ & 12 & 8 & $0.25903 \mathrm{D}-20$ & $0.103 D+02$ \\
\hline & & 10 & $0.41460 D+10$ & 15 & 15 & $0.38193 \mathrm{D}-13$ & $0.165 \mathrm{D}+02$ & 22 & 14 & $0.26198 \mathrm{D}-19$ & $0.196 \mathrm{D}+02$ \\
\hline & & 100 & $0.40591 D+14$ & 21 & 21 & $0.61171 \mathrm{D}-14$ & $0.236 \mathrm{D}+02$ & 33 & 20 & $0.17403 \mathrm{D}-17$ & $0.285 \mathrm{D}+02$ \\
\hline
\end{tabular}

Table A-5: Results of the rank $n-1$ test problems from the CUTE collection

\begin{tabular}{|c|c|c|c|c|c|c|c|c|c|c|c|}
\hline \multirow[b]{2}{*}{ func } & \multirow[b]{2}{*}{$n$} & \multirow[b]{2}{*}{$x_{0}$} & \multirow[b]{2}{*}{ insts } & \multicolumn{4}{|c|}{ Standard } & \multicolumn{4}{|c|}{ Tensor } \\
\hline & & & & $f c n$ & grad & finalf & time & $f c n$ & grad & finalf & time \\
\hline \multirow[t]{2}{*}{ BRYBND } & 5000 & 1 & $0.12488 \mathrm{D}+06$ & 488 & 30 & 0.17586 D. 10 & $0.376 \mathrm{D}+03$ & 176 & 10 & $0.13179 \mathrm{D}-10$ & $0.130 D+03$ \\
\hline & & $\begin{array}{c}10 \\
100\end{array}$ & $\begin{array}{l}0.10763 D+12 \\
0.12303 D+18\end{array}$ & .3396 & 201 & $0.97750 \mathrm{D}-21$ & $0.263 \bar{D}+04$ & $\begin{array}{l}1088 \\
1560\end{array}$ & 84 & $0.16631 \mathrm{D}-11$ & $0.1110+04$ \\
\hline \multirow[t]{3}{*}{$D[X M A A N]$} & 3000 & 1 & $0.00000 \mathrm{D}+00$ & 1 & 1 & $0.00000 \mathrm{D}+00$ & 0.8000 .01 & 1 & 1 & $0.00000 \mathrm{D}+00$ & $0.700 \mathrm{D}-01$ \\
\hline & & 10 & $0.00000 D+00$ & 1 & 1 & $0.00000 D+00$ & $0.800 \mathrm{D}-01$ & 1 & 1 & $0.000000+00$ & $0.700 \mathrm{D}-01$ \\
\hline & & 100 & $0.000000+00$ & 1 & I & $0.00000 D+00$ & $0.700 \mathrm{D}-01$ & 1 & 1 & $0.00000 \mathrm{D}+00$ & $0.800 \mathrm{D}-01$ \\
\hline \multirow[t]{3}{*}{$D[X O N 3 D Q$} & 5000 & $t$ & $0.40000 D+01$ & 6 & 2 & $0.62536 \mathrm{D} \cdot 17$ & $0.712 \mathrm{D}+01$ & 6 & 2 & $0.62536 \mathrm{D}-17$ & $0.718 \mathrm{D}+01$ \\
\hline & & 10 & $0.12100 D+03$ & 6 & 2 & $0.18917 \mathrm{D} .15$ & $0.713 D+01$ & 6 & 2 & $0.18917 \mathrm{D}-15$ & $0.713 D+01$ \\
\hline & & 100 & $0.102010+0.5$ & 6 & 2 & $0.15948 \mathrm{D} .13$ & $0.713 D+01$ & 6 & 2 & $0.15948 \mathrm{D}-13$ & $0.713 D+01$ \\
\hline \multirow[t]{3}{*}{ NONDQUAR } & 10000 & 1 & $0.10003 D+0.5$ & - & IL & - & - & 182 & 24 & $0.57721 \mathrm{D}-07$ & $0.635 D+03$ \\
\hline & & 10 & $0.39981 D+08$ & - & IL & - & - & 4414 & 187 & D. $17004 \mathrm{D} .07$ & $0.608 D+04$ \\
\hline & & 100 & $0.39980 \mathrm{D}+12$ & - & IL & - & - & 3820 & 194 & $0.62846 \mathrm{D}-07$ & $0.560 \mathrm{D}+04$ \\
\hline \multirow[t]{3}{*}{ QUARTC } & 1000 & 1 & $0.45000 D+05$ & 57 & 15 & $0.61708 D .05$ & $0.631 D+01$ & 13 & 4 & $0.24654 \mathrm{D}-07$ & $0.144 D+01$ \\
\hline & & 10 & $0.45000 D+09$ & 81 & 21 & $0.366 .35 \mathrm{D}-0.5$ & $0.905 D+01$ & 29 & 5 & $0.53107 \mathrm{D}-07$ & $0.2400+01$ \\
\hline & & 100 & $0.45000 D+13$ & 101 & 26 & $0.110 .38 \mathrm{D} .04$ & $0.113 D+02$ & 130 & 22 & $0.50306 \mathrm{D} .06$ & $0.107 D+02$ \\
\hline \multirow[t]{3}{*}{ SHUSENBR } & 3000 & 1 & $0.48481 D+0.5$ & .30 & 8 & $0.15403 \mathrm{D}-03$ & $0.477 \mathrm{D}+02$ & 44 & 7 & $0.45822 \mathrm{D} .12$ & $0.422 \mathrm{D}+02$ \\
\hline & & 10 & $0.44888 D+10$ & 286 & 65 & $0.23622 \mathrm{D} .12$ & $0.440 D+0.3$ & 121 & 21 & 0.16 .5870 .10 & $0.146 D+03$ \\
\hline & & 100 & $0.51122 \mathrm{D}+14$ & - & IL & - & - & 242 & 49 & $0.35217 \mathrm{D}-11$ & $0.344 D+0.3$ \\
\hline \multirow{3}{*}{ TQUARTIC } & 1000 & 1 & $0.32368 \mathrm{D}+04$ & 38 & 12 & $0.38436 D .15$ & $0.4 .33 D+01$ & 17 & 4 & $0.98215 \mathrm{D}-17$ & $0.15 .5 D+01$ \\
\hline & & 10 & $0.15962 D-23$ & 1 & 1 & $0.98215 \mathrm{D}-17$ & $0.200 \mathrm{D}-01$ & 1 & I & $0.38215 \mathrm{D}-17$ & $0.200 \mathrm{D}-01$ \\
\hline & & 100 & $0.32368 \mathrm{D}+06$ & 23 & 8 & $0.20695 \mathrm{D} .13$ & $0.2750+01$ & 28 & 9 & $0.14036 \mathrm{D}-15$ & $0.335 \mathrm{D}+01$ \\
\hline \multirow[t]{3}{*}{ TRIDIA } & 10000 & $i$ & $0.50005 \mathrm{D}+08$ & 6 & 2 & $0: 4115.5 \mathrm{D}-14$ & $0.267 \mathrm{D}+02$ & 6 & 2 & $0.4115 .5 \mathrm{D}-14$ & $0.266 \mathrm{D}+02$ \\
\hline & & 10 & $0.50005 D+10$ & 6 & 2 & $0.44399 \mathrm{D} \cdot 12$ & $0.266 \mathrm{D}+02$ & 6 & 2 & $0.44999 \mathrm{D}-12$ & $0.2660+02$ \\
\hline & & 100 & $0.30005 D+12$ & 11 & 3 & $0.14577 \mathrm{D}-13$ & $0.531 D+02$ & 11 & 3 & o. $14914 \mathrm{D}-13$ & $0.535 D+02$ \\
\hline \multirow[t]{3}{*}{ wistos } & 1000 & 1 & $0.27296 \mathrm{D}+07$ & 248 & 49 & $0.52712 \mathrm{D}-11$ & $0.236 \mathrm{D}+02$ & 224 & 32 & $0.41898 \mathrm{D} \cdot 10$ & $0.168 D+02$ \\
\hline & & 10 & $0.22566 \mathrm{D}+11$ & 342 & 67 & $0.635340-11$ & $0.324 D+02$ & 245 & 38 & $0.20790 \mathrm{D}-11$ & $0.190 D+02$ \\
\hline & & 100 & $0.22122 \mathrm{D}+1.5$ & 446 & 87 & $0.44137 D-11$ & $0.423 D+02$ & 308 & 47 & $0.22064 \mathrm{D}-10$ & $0.247 D+02$ \\
\hline \multirow[t]{3}{*}{ WOODS1 } & 1000 & 1 & $0.55491 \mathrm{D}+0.5$ & 86 & 18 & 0.252010 .03 & $0.816 D+01$ & 50 & 10 & $0.219810-08$ & $0.463 D+01$ \\
\hline & & 10 & $0.41460 D+09$ & 116 & 24 & $0.21634 \mathrm{D}-09$ & $0.1110+02$ & 84 & 16 & $0.40452 \mathrm{D}-08$ & $0.765 D+01$ \\
\hline & & 100 & $0.405010+13$ & 146 & 30 & $0.13591 \mathrm{D}-09$ & $0.1330+02$ & 125 & 22 & $0.50008 \mathrm{D}-08$ & $0.108 D+02$ \\
\hline
\end{tabular}


Table A-6: Results of the rank $n-2$ test problems from the CUTE collection

\begin{tabular}{|c|c|c|c|c|c|c|c|c|c|c|c|}
\hline \multirow[b]{2}{*}{ func } & \multirow[b]{2}{*}{$n$} & \multirow[b]{2}{*}{$x_{0}$} & \multirow[b]{2}{*}{ initf } & \multicolumn{4}{|c|}{ Standard } & \multicolumn{4}{|c|}{ Tensor } \\
\hline & & & & $\mathrm{fch}_{\mathrm{Cn}}$ & grad & linalf & time & $f c n$ & grad & finalf & lime \\
\hline \multirow[t]{3}{*}{ BRYBND } & 3000 & 1 & $0.12487 D+06$ & 327 & 29 & $0.42337 \mathrm{D}-09$ & $0.454 \mathrm{D}+03$ & 268 & 14 & $0.30203 \mathrm{D}-08$ & $0.219 \mathrm{D}+03$ \\
\hline & & 10 & $0.10765 D+12$ & 824 & 46 & $0.16732 \mathrm{D}-15$ & $0.724 D+03$ & 670 & 32 & $0.34308 \mathrm{D}-10$ & $0.519 D+03$ \\
\hline & & 100 & $0.12303 D+18$ & - & IL & - & - & 1401 & 68 & $0.26897 \mathrm{D}-12$ & $0.1100+04$ \\
\hline \multirow[t]{3}{*}{ DIXMAANS } & 3000 & 1 & $0.00000 D+00$ & $\mathfrak{l}$ & 1 & $0.00000 D+00$ & $0.800 \mathrm{D} .01$ & 1 & 1 & $0.00000 D+00$ & 0.7000 .01 \\
\hline & & 10 & $0.00000 D+00$ & 1 & 1 & $0.00000 \mathrm{D}+00$ & $0.800 D .01$ & I & 1 & $0.00000 \mathrm{D}+00$ & $0.800 D-01$ \\
\hline & & 100 & $0.000000+00$ & 1 & 1 & $0.00000 \mathrm{D}+00$ & $0.800 \mathrm{D}-01$ & 1 & 1 & $0.00000 \mathrm{D}+00$ & $0.8000-01$ \\
\hline \multirow[t]{3}{*}{$D[X \cup N 3 D Q$} & 5000 & 1 & $0.80000 D+01$ & 7 & 2 & $0.62564 \mathrm{D}-17$ & $0.938 D+01$ & 7 & 2 & $0.62564 \mathrm{D}-17$ & $0.938 D+01$ \\
\hline & & 10 & $0.24200 D+03$ & 7 & 2 & $0.18928 \mathrm{D} .15$ & $0.934 D+01$ & 7 & 2 & $0.18928 \mathrm{D}-15$ & $0.934 D+01$ \\
\hline & & 100 & $0.20402 D+05$ & 7 & 2 & $0.15948 \mathrm{D}-13$ & $0.933 D+01$ & 7 & 2 & $0.15948 \mathrm{D}-13$ & $0.936 \mathrm{D}+01$ \\
\hline \multirow[t]{3}{*}{ NONDQUAR } & 10000 & 1 & $0.10002 D+05$ & - & IL & - & - & 1109 & 70 & $0.14468 \mathrm{D}-06$ & $0.271 D+04$ \\
\hline & & 10 & $0.99980 D+08$ & - & IL & - & - & 1674 & 86 & $0.96220 \mathrm{D} .07$ & $0.332 \mathrm{D}+04$ \\
\hline & & 100 & $0.99980 D+12$ & - & IL & - & - & $1923^{-}$ & 101 & $0.40263 \mathrm{D}-07$ & $0.382 \mathrm{D}+04$ \\
\hline \multirow[t]{3}{*}{ QUARTC } & 1000 & 1 & $0.45000 D+05$ & 57 & 15 & $0.61708 \mathrm{D}-05$ & $0.646 \mathrm{D}+01$ & 13 & 4 & 0.24654 D- 07 & $0.145 D+01$ \\
\hline & & 10 & $0.45000 D+09$ & 81 & 21 & $0.36635 \mathrm{D}-05$ & $0.921 D+01$ & 101 & 17 & $0.53107 \mathrm{D}-07$ & $0.819 D+01$ \\
\hline & & 100 & $0.45000 D+13$ & 101 & 26 & $0.11038 \mathrm{D}-04$ & $0.115 D+02$ & 130 & 22 & $0.50906 \mathrm{D}=06$ & $0.107 D+02$ \\
\hline \multirow[t]{3}{*}{ SROSENBR } & 5000 & 1 & $0.484810+05$ & 72 & 13 & $0.82242 \mathrm{D}-14$ & $0.108 \mathrm{D}+03$ & 91 & 15 & $0.23908 \mathrm{D} \cdot 16$ & $0.128 D+03$ \\
\hline & & 10 & $0.44890 D+10$ & 429 & 77 & $0.69440 \mathrm{D}-04$ & $0.683 D+03$ & 465 & 68 & $0.14337 \mathrm{D}-16$ & $0.615 \mathrm{D}+03$ \\
\hline & & 100 & $0.51122 \mathrm{D}+14$ & - & IL & - & - & 1294 & 201 & $0.80433 D+06$ & $0.183 \mathrm{D}+04$ \\
\hline \multirow[t]{3}{*}{ TQUAATIC. } & 1000 & 1 & $0.32335 \mathrm{D}+04$ & 48 & 12 & $0.346 .35 \mathrm{D}-16$ & $0.565 D+01$ & 30 & 6 & $0.65443 D-18$ & $0.305 \mathrm{D}+01$ \\
\hline & & 10 & $0.15946 \mathrm{D}-23$ & 1 & 1 & $0.15946 \mathrm{D}-23$ & $0.200 \mathrm{D} .01$ & 1 & 1 & $0.15946 \mathrm{D}-23$ & $0.200 \mathrm{D}-01$ \\
\hline & & 100 & $0.32335 \mathrm{D}+06$ & 49 & 12 & 0.18893 D. 15 & $0.564 D+01$ & 54 & 12 & $0.56162 \mathrm{D} \cdot 18$ & $0.636 \mathrm{D}+0 \mathrm{t}$ \\
\hline \multirow[t]{3}{*}{ TRIDIA } & 10000 & 1 & $0.50005 \mathrm{D}+08$ & 8 & 2 & $0.41344 \mathrm{D}-14$ & $0.349 D+02$ & 8 & 2 & 0.41344 D. 14 & $0.349 D+02$ \\
\hline & & 10 & $0.50005 D+10$ & 8 & 2 & $0.45002 \mathrm{D} .12$ & $0.350 \mathrm{D}+02$ & 8 & 2 & $0.45002 \mathrm{D} .12$ & $0.349 D+02$ \\
\hline & & 100 & $0.50005 D+12$ & 15 & 3 & $0.25973 \mathrm{D}-12$ & $0.703 D+02$ & 15 & 3 & $0.25973 \mathrm{D} .12$ & $0.709 D+02$ \\
\hline \multirow[t]{3}{*}{ WOODS } & 1000 & 1 & $0.27277 \mathrm{D}+07$ & 196 & 31 & 0.77284 D- 1.3 & $0.189 D+02$ & 168 & 26 & $0.18453 \mathrm{D}-12$ & $0.165 \mathrm{D}+02$ \\
\hline & & 10 & $0.225610+11$ & 325 & 51 & $0.68702 \mathrm{D} .06$ & $0.316 \mathrm{D}+02$ & 289 & 41 & $0.10869 \mathrm{D}-12$ & $0.268 \mathrm{D}+02$ \\
\hline & & 100 & $0.221210+15$ & 434 & 68 & $0.56038 \mathrm{D}-05$ & $0.423 D+02$ & 89 & 11 & $0.11251 D .08$ & $0.6840+01$ \\
\hline \multirow[t]{3}{*}{ WOODSI } & 1000 & 1 & $0.55470 D+0.5$ & 118 & 18 & $0.18927 \mathrm{D}-09$ & $0.107 D+02$ & 91 & 16 & $0.10966 \mathrm{D} .07$ & $0.97 .5 D+01$ \\
\hline & & 10 & $0.41458 \mathrm{D}+09$ & - & $\mathrm{NC}$ & - & - & 127 & 22 & $0.30436 \mathrm{D} .08$ & $0.136 \mathrm{D}+02$ \\
\hline & & 100 & $0.405900+13$ & - & $\mathrm{NC}$ & - & - & 31 & 6 & $0.19654 \mathrm{D}-08$ & $0.324 \mathrm{D}+01$ \\
\hline
\end{tabular}

